\title{
Simultaneous discovery of candidate imprinted genes and Imprinting Control Regions in the mouse genome
}

\author{
Minou Bina* and Phillip Wyss \\ Department of Chemistry, Purdue University, West Lafayette, IN 47907, USA \\ ${ }^{*}$ Corresponding author bina@purdue.edu
}

\begin{abstract}
In mammals, parent-of-origin-specific gene expression is regulated by specific genomic DNA segments known as Imprinting Control Regions (ICRs) and germline Differentially Methylated Regions (gDMRs). In the mouse genome, the known ICRs/gDMRs often include clusters of a set of composite-DNA-elements known as ZFBS-morph overlaps. These elements consist of the ZFP57 binding site (ZFBS) overlapping a subset of the MLL1 morphemes. To improve detection of such clusters, we created density-plots. In genome-wide analyses, peaks in these plots pinpointed $\sim 90 \%$ of the known ICRs/gDMRs and located candidate ICRs within relatively long genomic DNA sections. In several cases, the candidate ICRs mapped to chromatin boundaries, to a subset of gene-transcripts, or to both. By viewing the plots at the UCSC genome browser, we could examine the candidate ICRs in the context of the genes in their vicinity. This strategy uncovered several potential imprinted genes with a broad range of physiologically important functions. Examples include: folliculogenesis; lineage commitment of murine embryonic stem cells; the development of the junctional zone of the placenta; left-right patterning of the body axis; the development of the neocortex, hippocampus, and cerebellum; postnatal vision; self-renewal of mouse spermatogonial stem cells; and histone-to-protamine replacement during spermatogenesis.
\end{abstract}

Predicted imprinted genes: Arid1b, Bcl2l1, Chd5, Cited2, Ccm2l, Cnnm1, Fam40b, Foxo6, Hectd1, Hivep2, Impdh1, Lsm14b, KRAZ1, Maml3, Mgea5, Nfix, Nkx2, Pou3f1, Pou3f3, Rev1, Six1, Slc35b4, Strip2, Stx7, Taf4a, t complex sterility, Zbtb2

\section{INTRODUCTION}

In mammals, the inherited pairs of chromosomes are not functionally identical [1-3]. In the course of gametogenesis, genomic imprinting affects a subset of genes and results in monoallelic, parental-of-origin specific expression $[4,5]$. The imprinting process is relatively complex. It involves chromatin remodeling, methylation of the ICRs/DMRs in one the parental alleles, and binding of ZFP57 to a subset of the modified CpGs to maintain the DNA methylation memory [6]. Among the DNA methylation systems, a protein-complex consisting of DNMT3A and DNMT3L plays a central role in genomic imprinting [6,7]. This complex methylates DNA processivity [8]. The rate of product formation is influenced by the number of $\mathrm{CpGs}$, particularly by those dispersed in the CpG islands (CGls) associated with gene promoters [8]. Within these islands are clusters of CpG-rich motifs known as the MLL1 morphemes [9]. The MT domain in MLL1 and a related family member (MLL2) binds CpG-rich motifs $[10,11]$. Both MLL1 and MLL2 function in promoter-specific trimethylation of histone H3 lysine 4 producing H3K4me3 marks in chromatin [12]. Besides gene promoters, the CpG islands also occur within intergenic and intragenic sequences $[9,13]$. Promoter-associated islands often extend into the transcribed sequences, even into protein-coding exons [14]. The occurrences of the MLL1 morphemes in these exons impact codon utilization and preservation [15], perhaps reflecting the importance of these CpG-rich motifs in genome-associated functions.

The fully characterized ICRs/gDMRs often include sequences known as ZFBS-morph overlaps [13, 16]. These composite-DNA-elements are CpG-rich and thus could facilitate the processive methylation of several CpGs by the DNMT3A-DNMT3L complex [16]. While in vitro ZFP57 binds methylated DNA [17], the MT domain in MLL1 and MLL2 selectively interacts with unmodified sequences [10,11]. Overall, CpG dinucleotides occur 
infrequently in animal DNA [18]. Thus, in mammalian DNA the hexameric ZFP57 binding site occurs less often than sequences that do not contain any CpG [17]. Moreover, since the composite DNA elements contain 2 or more CpGs, their occurrences in the DNA are far less than those observed for the canonical ZFP57 binding site [16]. Since clusters of 2 or more ZFBS-morph overlaps located $\sim 90 \%$ of the characterized ICRs/gDMRs in the mouse genome [17], we wished to explore whether locating such clusters could pinpoint candidate ICRs and reveal the genomic positions of potential imprinted genes. However, even though the ZFBS-morph overlaps represent only $\sim 12.7 \%$ of ZFP57 binding sites along Chr7 [16], their isolated occurrences create background noise. A noisy background would interfere with discerning the genomic positions of the ICRs/gDMRs within relatively long DNA sections. To resolve this problem, we devised a bioinformatics strategy. The scheme consisted of creating plots to display the density of ZFBS-morph overlaps in mouse genomic DNA. Previously, this approach facilitated locating clusters of ZFBS-morph overlaps in the human genome [19]. To explore the predictive capacity of our strategy for the mouse genome, we sampled the 'robust' peaks in the density-plots, to identify the genes or transcripts in their vicinity. The examined samples mapped to genes important to fetal growth, vision, organogenesis, neurogenesis, and sex-related processes.

\section{RESULTS}

We wrote a Perl script that employed a specified sliding window to determine and to report the density of ZFBSMorph overlaps along chromosomal DNA sequences. We chose the window-size (the number of covered DNA bases) by trial and error. To do so, we selected the extensively characterized ICR of the H19 - lgf2 imprinted domain to gauge the appearance of peaks in the density-plots. We noticed that long windows gave spurious peaks; small windows had a spikey appearance. Based on this and related exploratory studies, we chose a window consisting of 850 bases. For this window-size, the ICR of the H19 - Igf2 imprinted domain appears under two density peaks (Fig. 1). This ICR includes several chromatin boundaries [20, 21], and two ZFP57 associated regions [22]. The locations of the two density peaks agree with positions of ZFP57associated regions and the chromatin boundaries within the ICR of the H19 - Igf2 imprinted domain.

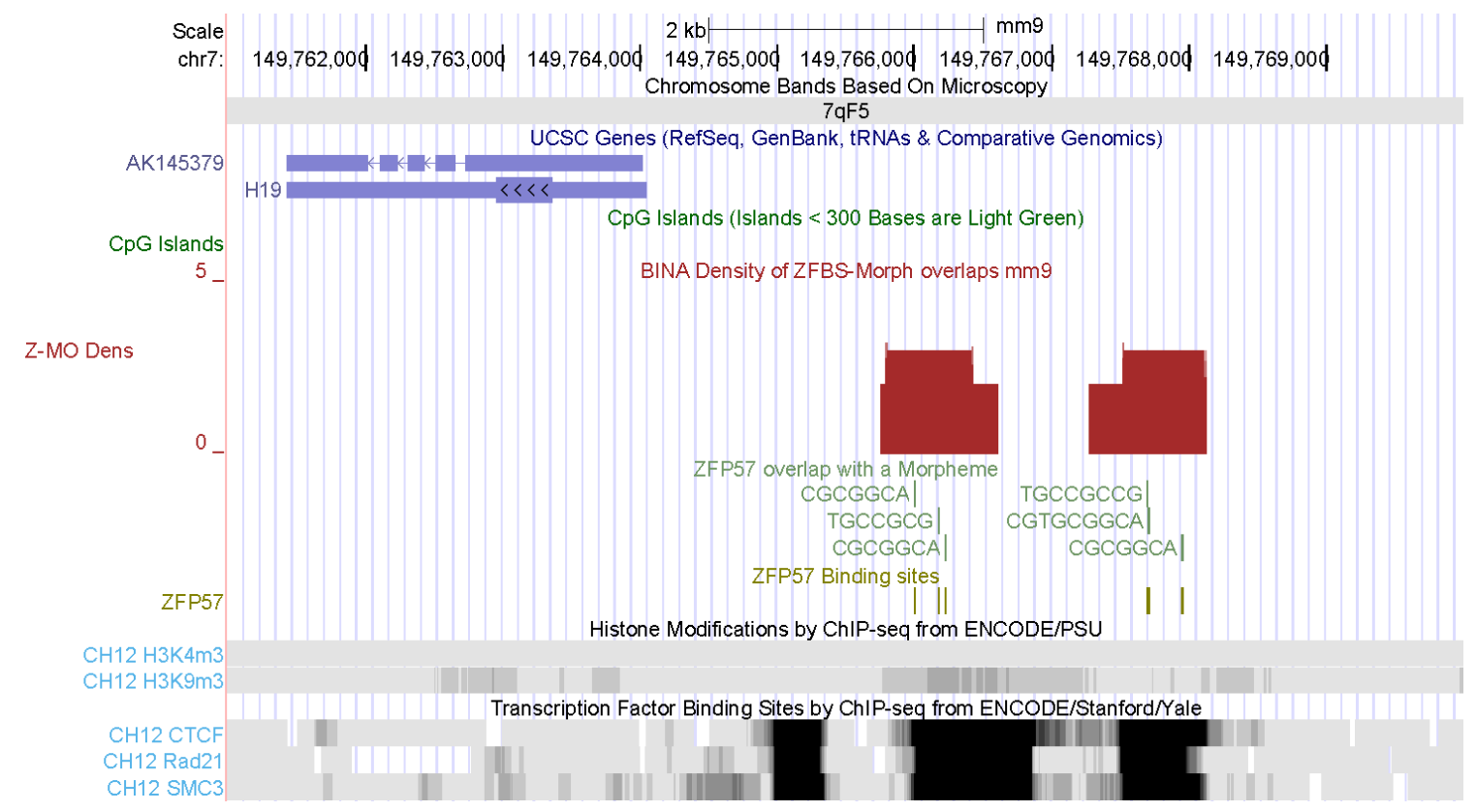

Figure 1. Genomic position of the ICR in the H19 - lgf2 imprinted domain in the 850-base window selected for creating the density-plots. Note that the ICR does not include H3K9me3 marks. This is because with ChIPs, occasionally the antibody cannot detect highly condensed regions in chromatin. 
In preliminary assessments, we found that density peaks covering 2 ZFBS-Morph overlaps could be false or true-positive. Therefore, to locate candidate ICRs, we focused on the 'robust' peaks encompassing 3 or more ZFBS-Morph overlaps. In initial evaluations, we asked whether we could spot the known ICRs/gDMRs within relatively long genomic DNA sections. Concurrently, we inspected these DNA sections for peaks that may reflect the genomic positions of candidate ICRs. By displaying the density-plots at the UCSC browser, we could obtain close-up views to investigate the positions of the candidate ICRs with respect to genomic landmarks; including genes, transcripts, and the CpG islands. We also sampled randomly selected DNA segments to determine whether they included chromatin boundaries, genes important to embryonic development, or both.

\section{Locating the position of ICRs/gDMRs in relatively long genomic DNA sections}

1. Density peaks revealed the essential ICR/gDMR of the complex Gnas locus and candidate ICRs within the Lsm14b and Taf4a loci

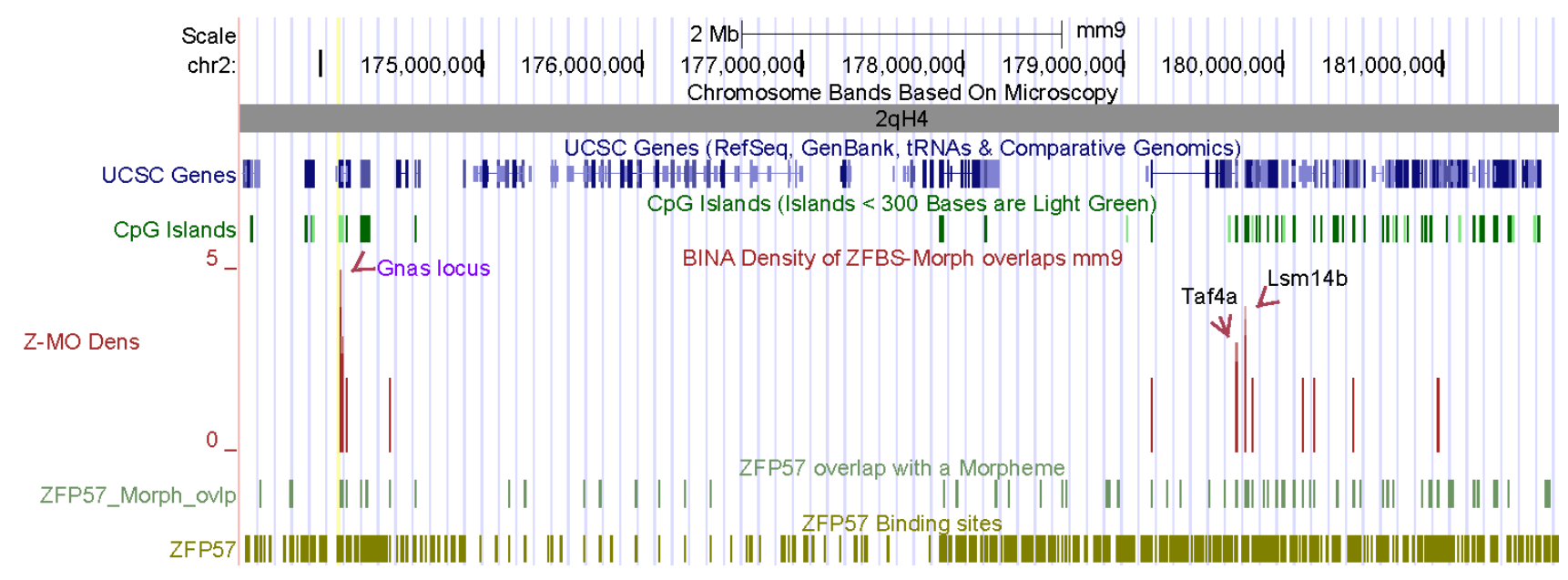

Figure 2. Genomic positions of density peaks along Chr2qH4. The displayed tracks give the locations of chromosomal bands (gray), genes (blue), the CpG islands (green), density peaks (maroon), the ZFBS-morph overlaps (hunter green), and the ZFP57 binding sites (olive). The density-plot is shown in full format. The remaining tracks are shown in dense format. An arrow points to the peak corresponding to the essential ICR of the Gnas complex locus (shown in purple). Two arrows point to candidate ICRs near the Taf4a and Lsm14b loci. Note that at such a low-resolution physical map (covering $\sim 8.25 \mathrm{Mb} D N A$ ), clearly resolved are density peaks covering 2 or more ZFBS-morph overlaps. In contrast, the positions of the CpG islands are not resolved. The occurrences of the hexameric ZFP57 site are not clearly apparent. Even though less frequent, there are also many isolated ZFBS-morph overlaps.

The Gnas complex locus is in Chr2qH4. This chromosomal band contains 8.25 Mb DNA (Fig. 2). Along the displayed segment, discernable are 3 robust density peaks and several peaks covering 2 ZFBS-Morph overlaps. Thus, for that DNA section we observed 1 robust peak per $\sim 2.57 \mathrm{Mb}$ DNA. One of the 3 robust peaks located the essential ICR of the complex Gnas locus. The other 2 correspond to candidate ICRs (Fig. 2). In enlarged views, the peak in the Gnas locus appeared as a doublet defining the two distinguishable clusters of ZFBS-Morph overlaps in the locus $[13,16]$. In the DNA, a candidate ICRs maps to Taf4a (a subunit of the general transcription factor TFIID). Another one maps to Lsm14b (LSM family member 14B). From Lsm14b are produced three transcripts with differing exon utilization patterns (Fig. S1). The density peak is in the CGI that encompasses the promoter and the TSS of the longest $L s m 14 b$ transcript (Fig. S1). Functionally, LSM14B is an RNA binding protein 
that plays an essential role in oocyte meiotic maturation through regulating mRNA pools [23]. For Taf4a, genomic maps also revealed several transcripts. The candidate ICR maps to the $1^{\text {st }}$ exon of the longest Taf4a transcript (Fig. S1). The corresponding density peak is between two $\mathrm{CpG}$ islands. One of the islands encompasses a bidirectional-promoter regulating expression from Taf4a and 4921531C22Rik. According to GENE at NCBI, 4921531C22Rik is broadly expressed in adult testes (https://www.ncbi.nlm.nih.gov/gene/66730\#geneexpression). Since the TSSs of Taf4a and 4921531C22Rik are not far-apart (Fig. S1), the candidate ICR may regulate allele-specific expression of both 4921531 C22Rik and the longest Taf4a transcript.

\section{Density peaks pinpointed the ICR/gDMR of Mest and revealed candidate ICRs for potential imprinted genes}

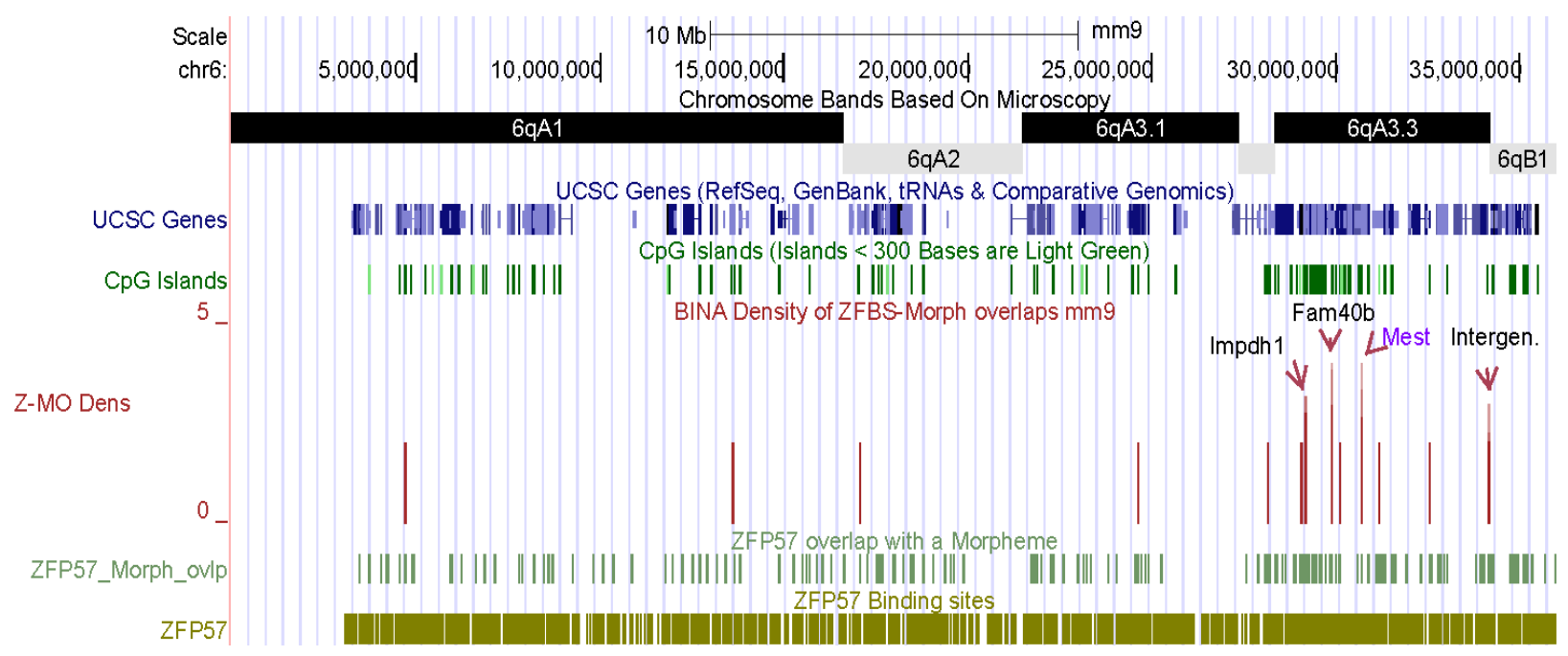

Figure 3. Genomic positions of robust density peaks along Chr6qA. One peak spotted the known ICR/gDMR of the Mest locus. Several peaks mark the position of candidate ICRs regulating expression of potential imprinted genes.

The ICR of Mest is in Chr6qA. This chromosomal band consists of several subbands. The entire band encompasses nearly $35 \mathrm{Mb}$ long DNA and 4 robust density peaks (Fig. 3). Thus, for that DNA section we observed 1 robust peak per $\sim 8.7 \mathrm{Mb}$ DNA. We noticed that overall, robust peaks were not uniformly distributed along the chromosomal DNA sequences. For example, 4 consecutive subbands (6qA1, 6qA2, 6qA3.1, 6qA3.2) contain only peaks covering 2 ZFBS-Morph-overlaps. In contrast, 6qA3.3 encompasses the 4 robust density peaks (Fig. 3). One of the peaks spotted the known ICR/gDMR in the Mest locus. The other 3 correspond to candidate ICRs. They map to: (i) a CGI that encompasses the TSS of Impdh1 (Fig. S2); (ii) a CGI that that encompasses the TSS of Fam4Ob (Fig. S3); and (iii) a region far upstream of the TSS of S/c35b4 (Fig. S4).

Functionally, IMPDH1 (inosine-5'-monophosphate dehydrogenase 1) catalyzes the rate-limiting step in biosynthesis of guanine nucleotides. Mutations in the corresponding human gene caused retinitis pigmentosa, an inherited retinal degeneration characterized by the early onset of night blindness followed by a progressive loss of the visual field [24, 25]. Also known as Strip2 (striatin interacting protein 2), Fam40b plays an indispensable role in the onset of embryonic stem cells (ESCs) differentiation [26]. SLC35B4 (solute carrier family 35, member B4) regulates obesity and glucose homeostasis [27]. 


\section{Density peaks located the ICR/gDMR of Zac1 and candidate ICRs for several potential imprinted genes}

The ICR of Zac1 is in Chr10qA. This chromosomal band covers 4 subbands and contains $34 \mathrm{Mb}$ DNA. For that DNA section, we observed 7 robust peaks $(1$ per $\sim 4.8 \mathrm{Mb}$ ). The Plag/1 locus includes an intragenic conserved CGI near one of the Plagl1 transcripts known as Zac1. In oocytes, this CGI is methylated and regulates parent-of-origin specific gene expression [28]. A previous study found a large cluster of ZFBS-Morph overlaps in the Zac1 ICR/gDMR [13]. The density-plot revealed a very robust density peak that encompasses that cluster in the intragenic CGI that corresponds to the Zac1 ICR/gDMR (Fig. S5). In subband 10qA1, a candidate ICR includes the promoter and the TSS of the longest Zbtb2 transcript (Fig. S6). ZBTB2 (Zinc Finger and BTB domain containing 2) is a transcription factor that is 'a reader' of unmethylated CpG island promoters to regulate ES cells differentiation in mice [29]. Subband 10qA3 covers an intergenic candidate ICR between the TSSs of Nhs/1 and Ccdc28a (Fig. S7). According to GENE at NCBI, Ccdc28a (coiled-coil domain containing 28A) is expressed in adult testis (https://www.ncbi.nlm.nih.gov/gene/215814\#gene-expression). The function Nhs/1 (NHS like 1) is not clearly understood. Subband 10qA4 includes a candidate ICR in a CGI that encompasses the TSSs of Stx7 transcripts (Fig. S8). Syntaxin-7 is among a group of proteins that function in vesicle transport and fusion events [30].

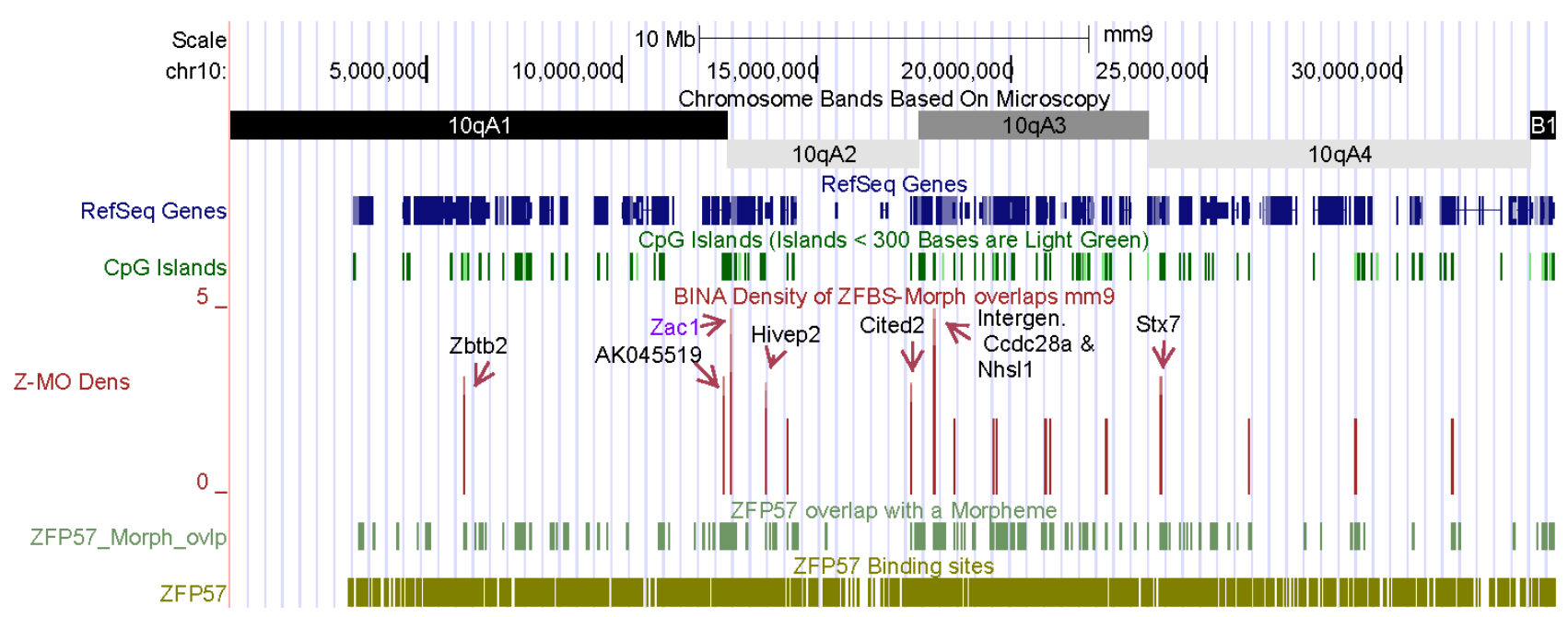

Figure 4. A long DNA section encompassing several candidate ICRs and the known ICR/gDMR of Zac1. A robust density peak pinpointed the known ICR/gDMR of Zac1 in $\sim 34 \mathrm{Mb}$ long DNA. Several additional robust peaks mark the positions of candidate ICRs for potential imprinted genes.

Besides the known ICR/gDMR of Zac1, subband 10qA2 includes 3 candidate ICRs (Fig. 4). They map to Cited2 (Fig. S9), to a CGI associated with several Hivep2 transcripts (Fig. S10), and to an uncharacterized gene (AK045519). Hivep2 (human immunodeficiency virus type I enhancer-binding protein 2) encodes a transcription factor that binds DNA [31]. In mice, Hivep2 deficiency Induced mild chronic inflammation in the brain and conferred molecular, neuronal, and behavioral phenotypes related to schizophrenia [32]. Cited2 (CBP/p300interacting transactivator 2) also is involved in the regulation of transcription. Absence of Cited 2 in mouse embryos caused congenital heart disease by perturbing left-right patterning of the body axis [33]. 
4. Density peaks revealed the known ICR/gDMR of Igf2r - Airn imprinted domain, and candidate ICRs mapping to Arid1b and to the tctex-1 locus (a candidate gene family for a mouse $t$ complex sterility)

In evaluations, we closely inspected the density-plot for every mouse autosomal chromosome. For example, examine the positions of peaks in the plot that covers the entire Chr17 (Fig. 5). Even along the entire chromosomal DNA ( 95.3 Mb), we could detect the robust density peak that corresponded to ICR of the Igf2r - Airn imprinted domain. Located in the $2^{\text {nd }}$ intron of $\operatorname{lgf} 2 r$, the ICR/gDMR of the domain regulates parent-of-origin specific expression of several genes [34]. This intronic ICR/gDMR is in a CGI in the vicinity of Airn promoter [16]. Also known as Air, Airn is the only paternally expressed gene in that imprinted domain. Transcription of Airn produces a noncoding RNA that is antisense orientation with respect to lgf2r and Mas [35]. In the density-plots, a very robust density peak maps to the intragenic $C G$ that regulate the expression of genes in the imprinted domain (Fig. S11). This observation supports the hypothesis that robust density peaks could correspond to the ICRs in the mouse genome.

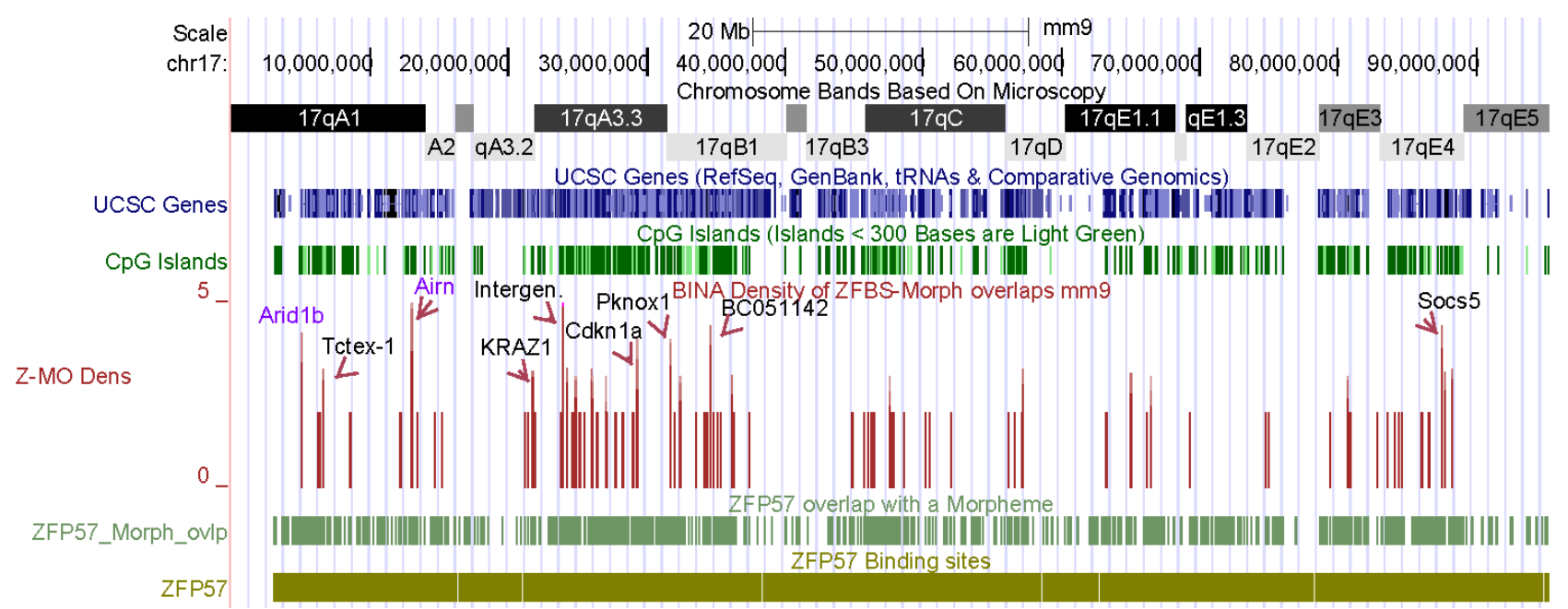

Figure 5. The position of peaks in the density-plot of Chr17. By uploading our data onto the UCSC genome browser, we could view the positions of density peaks across an entire chromosome. Peak-heights reflect the number of ZFBS-morph overlaps within the sliding 850-base window. A large fraction of peaks corresponds to those covering 2 ZFBS-morph overlaps. Robust peaks are less frequent and distributed unevenly along the DNA. In many regions, they appear as clusters - possibly a reflection of the intricacies of epigenetic regulation in clusters [36]. In exploratory evaluations, we encountered instances where 2 closely spaced peaks produced a robust density peak.

Key features of the genome browser include the option of uploading files to create custom tracks, as shown in this report, and to enhance the resolution of peaks by zooming in to obtain enlarged or close up views [37-39]. For example, an enlarged view shows the position of density peaks in a DNA section that covers the subbands in Chr17qA (Fig. 6). The displayed view covers $\sim 23 \mathrm{Mb}$ DNA and 4 robust density peaks (1/5.75 Mb). One peak corresponds to the known ICR/gDMR of the Igf2r - Airn imprinted domain. Three peaks mark the positions of candidate ICRs. They are in the vicinity of Arid1b, KRAZ1, and tctex-1 loci (Fig. 6). We chose that section because it includes the ICR/gDMR of the Igf2r - Airn imprinted domain and a candidate ICR for Arid $1 b$ -a known imprinted gene in mouse [40]. A robust density peak maps to the longest Arid1b transcripts (Fig. 7). Arid1b (AT rich interactive domain 1B) encodes an enzyme (KDM5B) that removes methyl-marks from H3K4. Depletion of Arid1b in ES cells caused defects in gene expression programs [41]. 


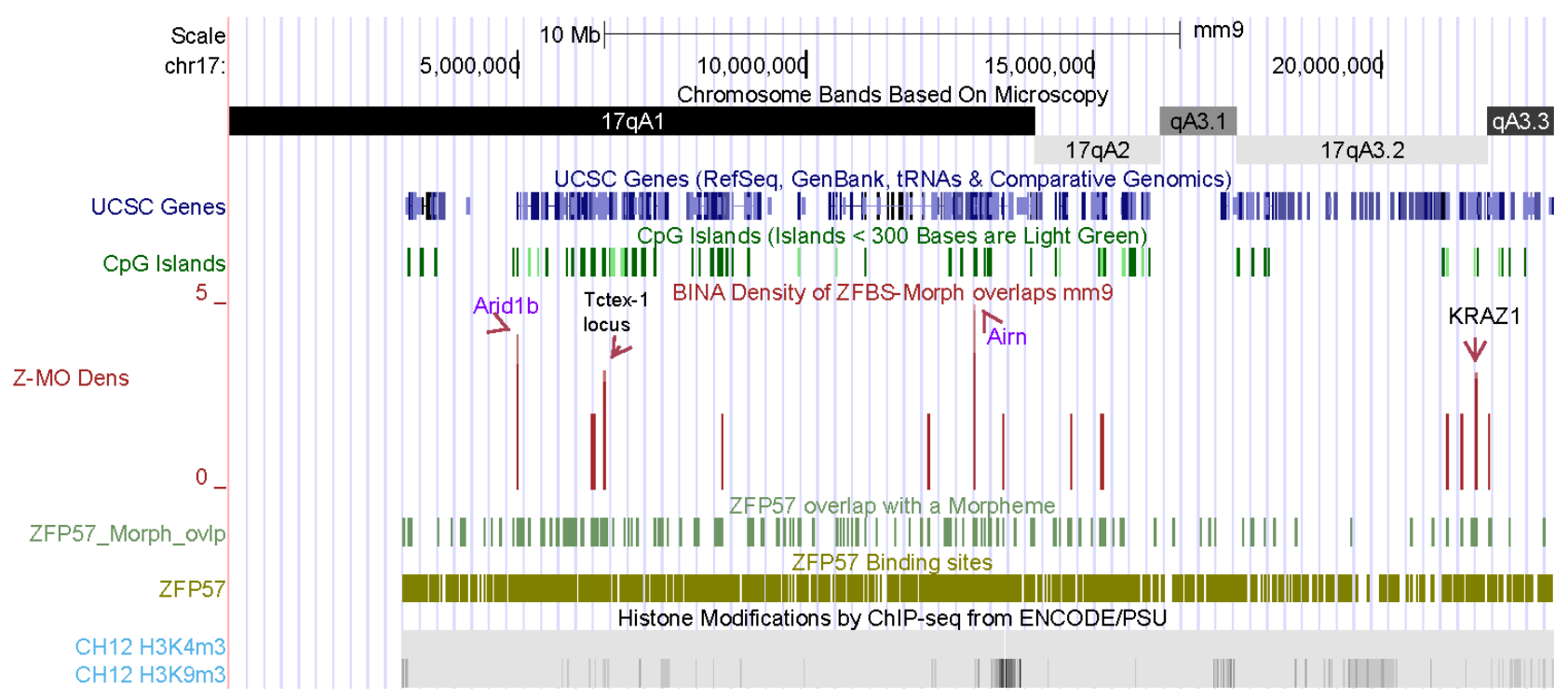

Figure 6. A view of the positions of the robust density peaks in the proximal end of Chr17. This chromosomal section includes candidate ICRs for Arid $1 b$ and potential imprinted loci.

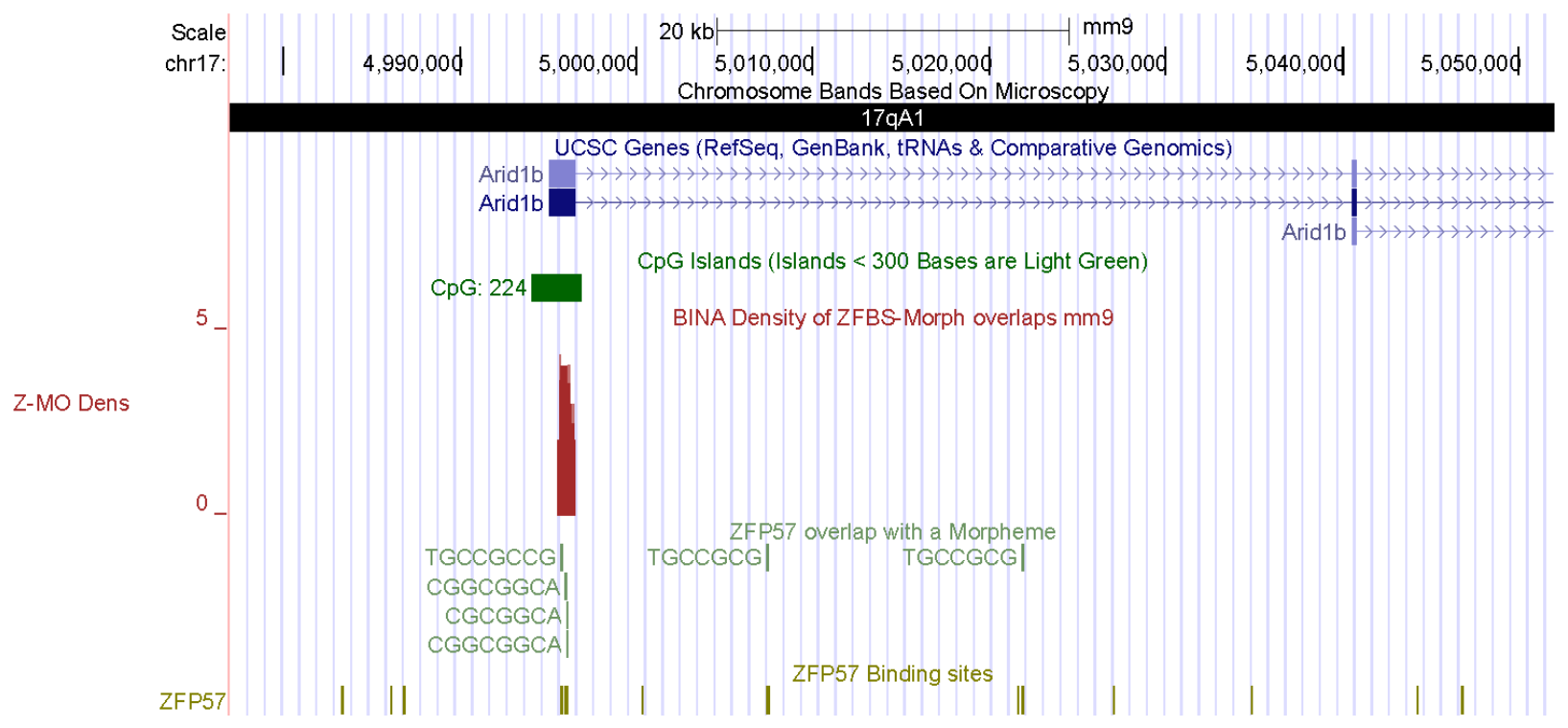

Figure 7. The position of a candidate ICR for Arid1b -a known imprinted gene in mouse [40].

In Chr17qA, another robust density peak is in the vicinity of KRAZ1 (Figs. 5, 6, and S12). KRAZ1 is in a chromosomal section that encompasses several genes for proteins whose structure includes zinc fingers (Fig. S12). KRAZ1 encodes a protein with Krüppel-type zinc finger structural motif also found in ZNF57, KRAZ2, and ZNF445; references [42-44]. As ZNF57, both KRAZ1 and KRAZ2 interacts with KAP1 [43]. Also known asTRIM28, KAP1 plays a central role in silencing of the imprinted genes [6].

Notably, Chr17qA is in the chromosomal section that includes naturally occurring variants known as thaplotypes [45]. The variants in thaplotypes are alleles of genes that exist as a polymorphism in wild mouse populations 
[46]. While males heterozygous for a t haplotype are normal, homozygous males are sterile [46, 47]. All $t$ haplotypes contain rearranged segments of DNA known as the t complex [45]. The aberrant expression of tctex1 is solely dependent on the thaplotype genes and occurs only in germ cells [45]. The tctex-1 locus consists of a four member multigene [48]. In males, the wild-type tctex-1 transcript was first abundantly expressed at the pachytene stage of meiosis and persisted throughout spermatogenesis [48]. In the density plot of Chr17, a robust density peak is in the vicinity of tctex-1 (Figs. 5 and 6). Members of this gene family includes Dynlt 1 (dynein light chain Tctex type 1). Dyneins are large multicomponent microtubule-based molecular motors involved in many fundamental cellular processes including mitosis [49]. In the tctex-1 locus, a candidate ICR maps to a CpG island at $\sim 800$ bps upstream of the TSS of one of the Dynlt1e genes/transcripts (marked by an arrow in Fig. 8). Thus, our finding predicts that the tctex-1 locus could be an imprinted domain regulated by an ICR defined by a robust density peak (Figs 5,6 , and 8 ).

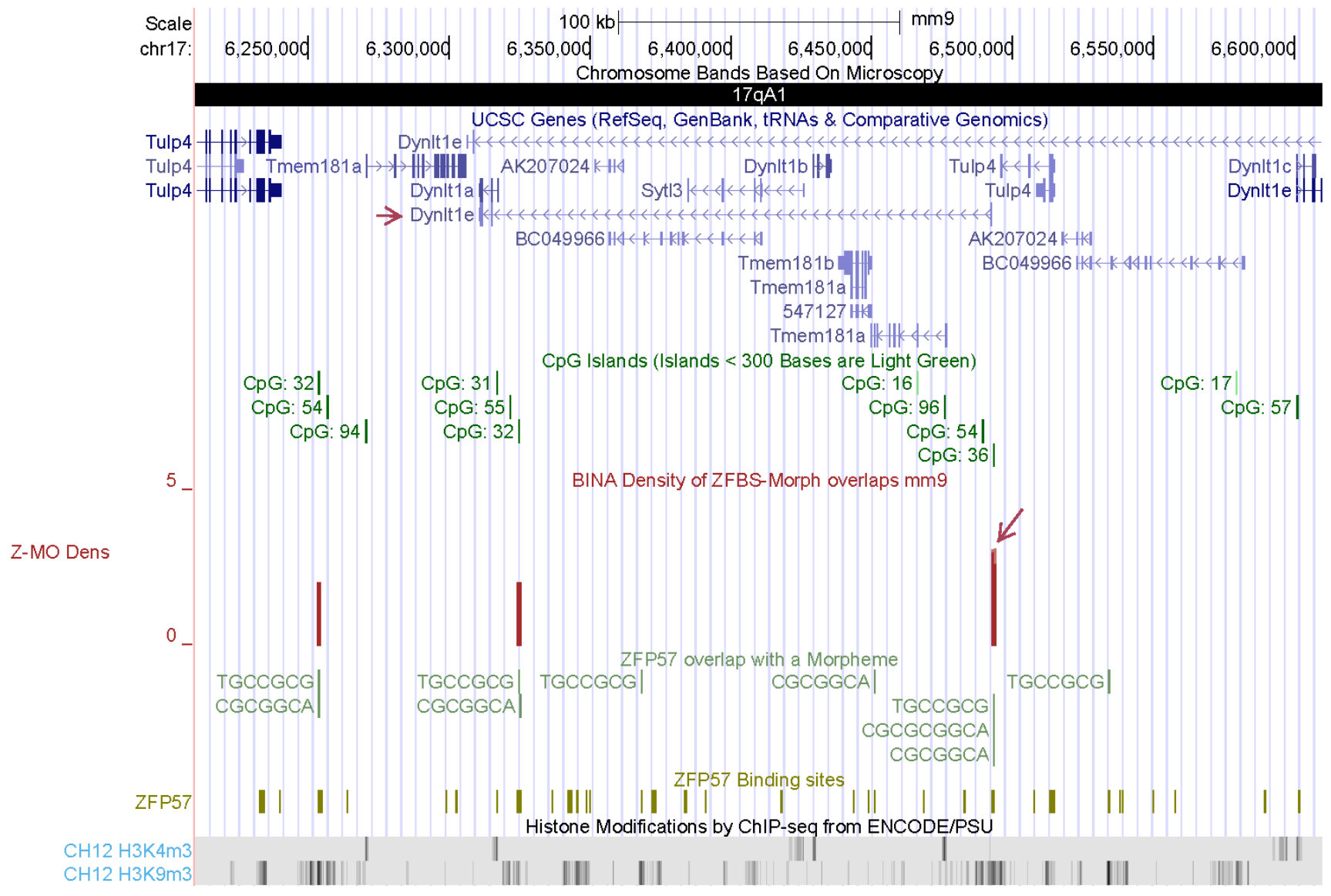

Figure 8. A candidate ICR mapping to the tctex-1 locus. An arrow points to the robust density peak providing a candidate ICR for regulation of expression from the tctex-1 locus. This peak is upstream of TSS of one the genes designated as Dynlt1e (marked by an arrow). Noteworthy are several patches of repressive H3K9me3 marks dispersed across the locus. These marks reinforce our prediction that the tctex-1 locus could be an imprinted domain. 
5. Density peaks revealed the known ICR/gDMR of Nnat, and candidate ICRs for Bc/2/1 and two potential imprinted genes

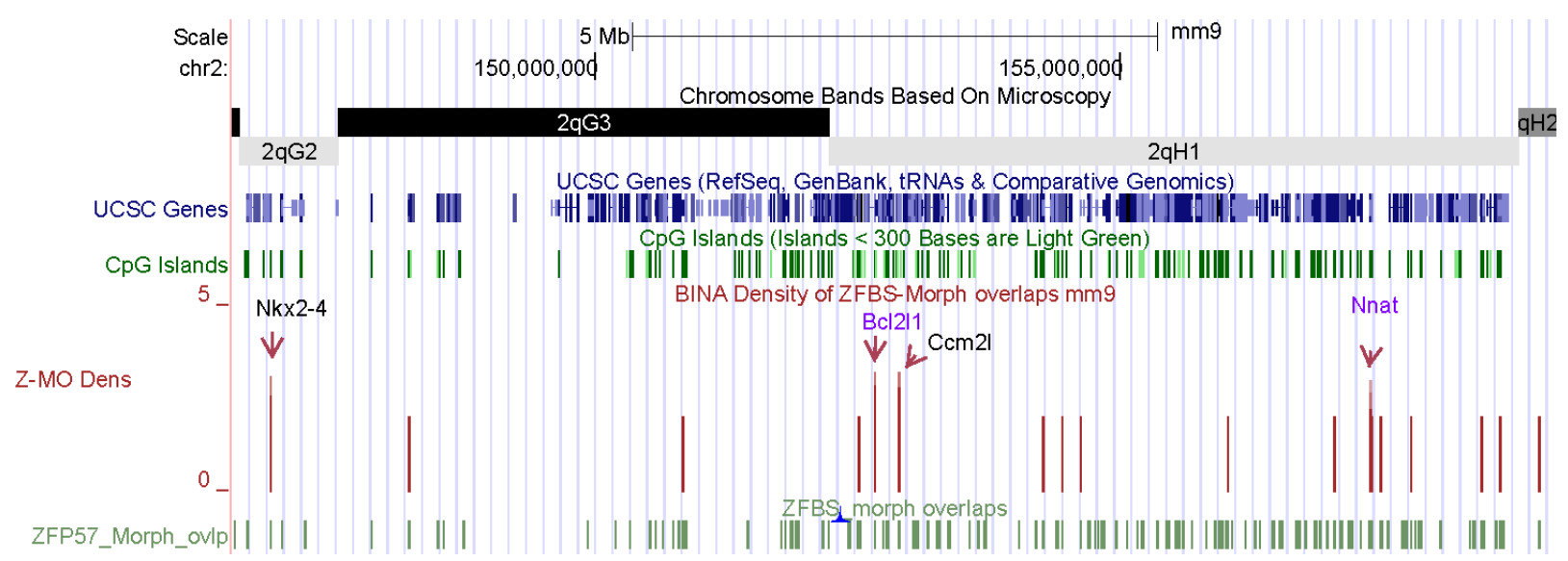

Figure 9. Genomic positions of density peaks along Chr2qG3 and $\mathrm{Chr} 2 \mathrm{qH} 1$. An arrow points to the peak that corresponds to the known ICR/gDMR of Nnat. Additional arrows mark the position of candidate ICRs.

In our exploratory analyses, we also examined an $\sim 12.6 \mathrm{Mb}$ DNA section encompassing Chr2qG3 and Chr2qH1 (Fig. 9). In that DNA section, we observed 4 robust density peaks (1 peak / 3.15 Mb). One peak pinpointed the ICR of Nnat. This ICR is in one of the introns of gene of a gene (Blcap) that is biallelically expressed [50, 51]. With respect to B/cap, Nnat is transcribed in the opposite direction. In transgenic animals, the ICR/gDMR of Nnat was mapped to a $\mathrm{CpG}$ island spanning the $1^{\text {st }}$ exon and extending into intron 2 [52]. According to genomic maps, from Nnat are produced several transcripts with differential exon utilization patterns (Fig. S13). In close-up views, we observed 2 peaks within a DNA segment that encompasses the intragenic Nnat ICR (Fig. S13). Besides the peak corresponding to the known, the $\sim 12.6 \mathrm{Mb}$ DNA includes 3 additional robust peaks. Two peaks are in $\mathrm{Chr} 2 \mathrm{qH} 1$. They are in the vicinity of $B c / 2 / 1$ and $\mathrm{Ccm} 2 \mathrm{l}$. The $3^{\text {rd }}$ peak maps to Chr2qG3, in the vicinity of Nkx2-4 (Fig. 9). In critical evaluations of imprinted gene expression by RNA-Seq, Bc/2/1 was listed as a candidate imprinted gene [53]. Furthermore, evidence supports the unequal contribution of the $B c / 2 / 1$ paternal and maternal alleles to brain development. Also referred to as $B c l-x$, haploinsufficiency of $B c / 2 / 1$ led to male-specific defects in fetal germ cells [54]. Notably, expression was paternally-biased for the longest $B c / 2 / 1$ isoform [55]. These findings support our prediction of a candidate ICR in the CGI that is associated with the longest Bc/2/1 transcript (Fig. 10). Furthermore, the predicted ICR is within chromatin boundaries consisting of CTCF, RAD21, and SMC3.

The candidate ICR for $\mathrm{Ccm} 2 /$ is intragenic and corresponds to a density peak is in the $5^{\text {th }}$ exon of the gene (Fig. S14). Ccm2I (cerebral cavernous malformation 2-like) is a paralog of Ccm2 [56]. CCM2 functions in the CCM signaling [57]. Its paralog (CCM2L) is selectively produced in endothelial cells during angiogenesis [57]. $\mathrm{Ccm} 2 \mathrm{I}^{-1-}$ animals exhibited embryonic lethality at E11 associated with myocardial thinning [56]. In Chr2qG3, a robust density peak maps to Nkx2-4 (Fig. 9). This candidate ICR is in a CpG island that encompasses Nkx2-4 TSS (Fig. S15). Notably, a chromatin section within Nkx2-4 includes repressive H3K9m3 marks. Nkx2-4 (NK2 homeobox 4) encodes a transcription factor whose structure includes a homeodomain for binding DNA [58]. During mouse embryogenesis, Nkx-2.2 transcripts were dispersed in localized domains possibly for specifying diencephalic neuromeric boundaries [58]. 


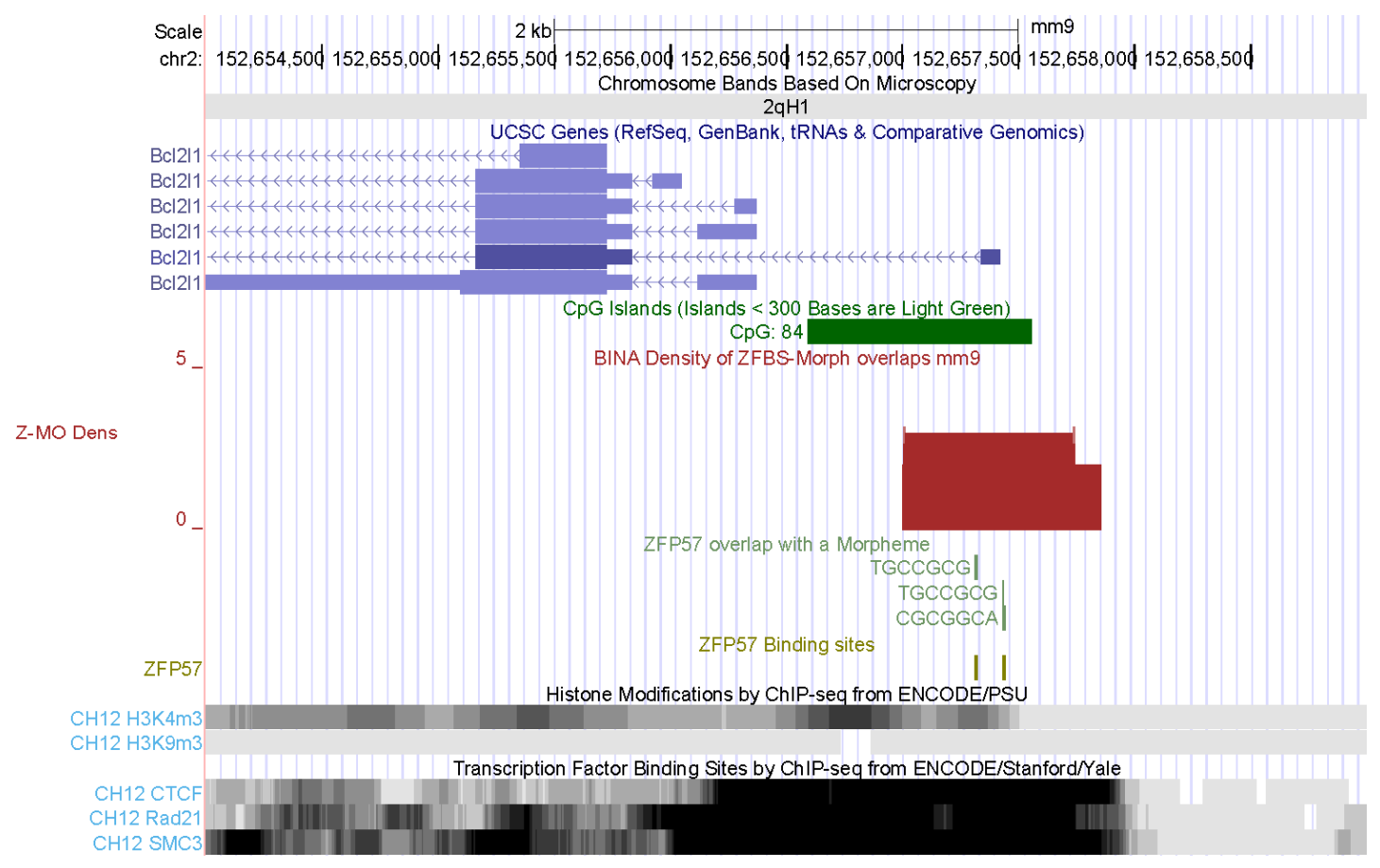

Figure 10. The position of a candidate ICR with respect to the longest $B c / 2 / 1$ transcript. Expression analyses have identified $B c / 2 / 1$ as a candidate imprinted gene [53].

\section{Several candidate ICRs are in the vicinity of chromatin boundaries}

It is well known that the ICR of the H19 - Igf2 imprinted domain includes chromatin boundaries [20, 21]. Furthermore, several of the known ICRs regulate a subset of gene-transcripts; i.e., Zac1, Inpp5f_v2, and Mest $[28,59,60]$. Therefore, to further evaluate our approach, we inspected the density-plots to determine whether any of the candidate ICRs mapped to specific gene-transcripts, to chromatin boundaries, or to both. For these studies, we surveyed the density-plots with respect to results of ChIPs obtained for CTCF, RAD21, and SMC3 reference [61]. Within the sampled DNA sections, we found several instances where a candidate ICR was associated with a single transcript and a chromatin boundary. Examples for a single transcript include a candidate ICR in a segment encompassing several members of the Six family (Fig. S16). The corresponding density peak is in a CGI that encompasses the TSS and the $1^{\text {st }}$ exon of Six1. It maps to a region that includes chromatin boundaries consisting of CTCF, RAD21, and SMC3 (Fig. S16). Six1 (sine oculis-related homeobox 1) encodes a transcription factor that plays central roles in the pre-placodal ectoderm, a common field from which arises all cranial sensory placodes including adenohypophyseal, olfactory, lens, trigeminal, epibranchial, and otic [62].

Examples of candidate ICRs mapping to a subset of gene transcripts include a density peak near the 5' end of the longest Hectd1 transcriptional variants (Fig. S17). This peak maps to a CGI that encompasses chromatin boundaries. Hectd1 (HECT domain E3 ubiquitin protein ligase 1) is required for development of the junctional zone of the placenta. Disruption of Hectd1 resulted in mid-gestation lethality and intrauterine growth restriction [63]. Another example is the candidate ICR that maps to the longest Bc/2/1 transcript (Fig. 10). Functionally, BCL2L1 belongs to a group of proteins related to BCL-2 (B cell leukemia/lymphoma 2). Members of this group control cell death through complex interactions that regulate mitochondrial outer membrane permeabilization. This process leads to the irreversible release of intermembrane space proteins with subsequent caspase activation and apoptosis [64]. 


\section{Several candidate ICR map to the vicinity of genes encoding transcription factors and proteins involved in sex-related processes}

Since transcription factors regulate the expression of many genes, they play central roles in numerous physiological processes. In humans, mutations in transcription factor genes could cause developmental abnormities know as syndromes [65]. As summarized above, near several of the candidate ICRs, we found genes encoding regulators of transcription (i.e. Taf4a, Zbtb2, Six1, Hivep2, Nkx2-4). Additional examples include Nfix, Mam/3, Pou3f3, Foxo6 and Pou3f1. Nfix is in Chr8qC3. Density-plots revealed a candidate ICR near the two longest Nfix transcripts (Fig. S18). Nfix (nuclear factor I/X) belongs to a group of transcription factors that play pivotal role in the development of the nervous system [66]. Mam/3 (mastermind like transcriptional coactivator 3) specifies a transcriptional coactivator. A candidate ICR is located in a CGI that encompasses the TSS of the longest Mam/3 transcript (Fig. S19). In vivo, Mam/3 and one of its paralogs (Mam/1) are essential for Notch signaling. This system plays a pivotal role in metazoan development [67]. Even though Mam/3-null mice showed no apparent abnormalities, mice null for both Mam/1 and Mam/3 died early in the organogenic period and displayed classic pan-Notch defects [67]. A relatively long DNA section ( $20 \mathrm{Mb})$ that encompasses Chr1qB includes only 2 robust density peaks ( $1 / 10 \mathrm{Mb})$. These 2 candidate ICRs are in the vicinity of Pou3f3 and Rev1 (Fig. 12). One is in a CGI that encompasses Pou3f3 TSS (Fig. S20). The displayed segment includes several repressive H3K9me3 marks. The other is in a CGI that encompasses Rev1 TSS (Fig. S21). This CGI is in the vicinity of chromatin boundaries. POU3F3 or BRN1 belongs to a group of transcription factors that influence neurogenesis, molecular identity, and migratory destination of upper-layer cells of the cerebral cortex [68]. Their structure incudes a POU-homeodomain for binding DNA [69].

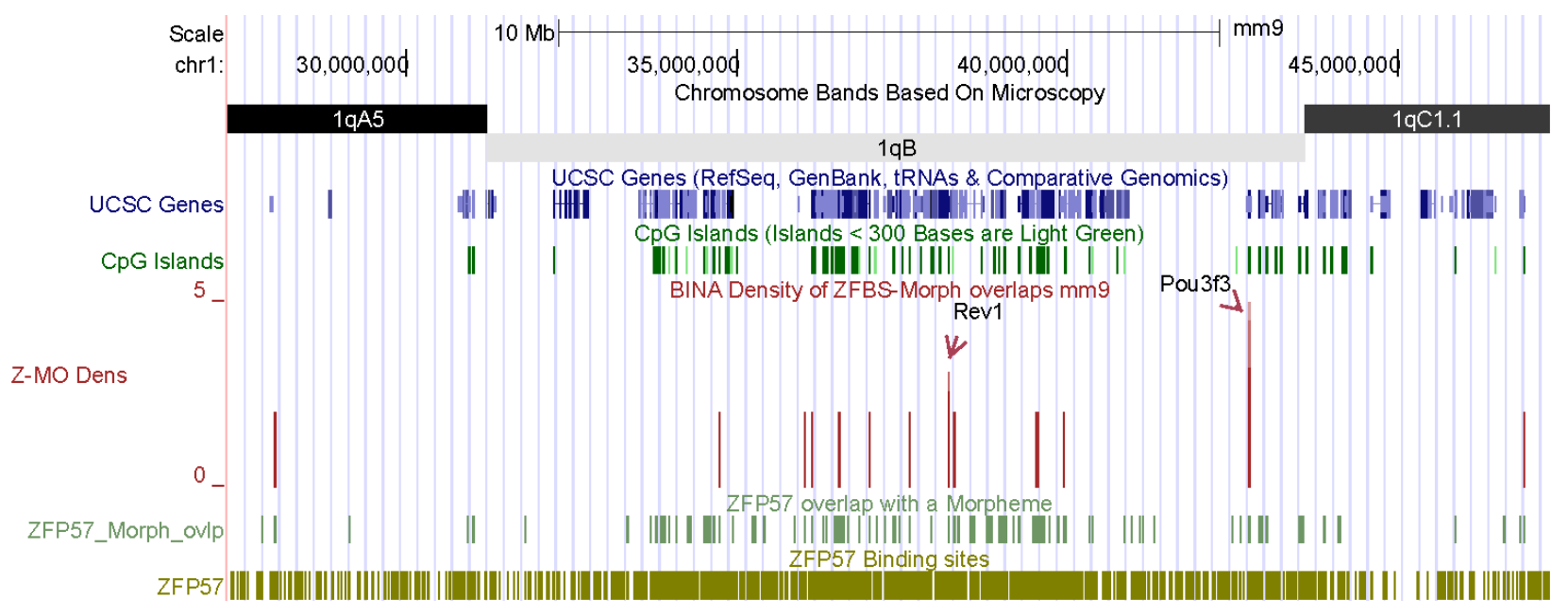

Figure 11. Two candidate ICRs within a relatively long DNA section. They are in the vicinity of Rev1 and Pou3f3.

Foxo6 and Pou3f1 are in Chr4qD2.2 (Fig. 12). The displayed DNA section ( 11.6 Mb) encompasses 2 robust density peaks (1 peak per $5.8 \mathrm{Mb}$ ). One peak gives a candidate ICR in a CGI that contains Foxo6 TSS and promotor (Fig. S22). The other corresponds to a candidate ICR in a CGI that encompasses Pou3f1 TSS (Fig. S21). The structure of FOXO6 includes a forkhead (winged helix) domain for binding cis elements in DNA [70]. That of POU3F1 includes a POU-homeodomain for interactions with DNA [69]. Even though both FOXO6 and POU3F1 are transcription factors, they play distinguishable roles in the regulation of gene expression. Foxo6 is expressed at late stages of face development [71]. Foxo6 ${ }^{-1-}$ mice underwent expansion of the face, frontal cortex, olfactory component and skull [71]. Pou3f1 plays a role in spermatogenesis [72]. Briefly, fertility of adult males 
requires self-renewal and differentiation of spermatogonial stem cells. A study found that in cells cultured in vitro, Pou3f1 expression was up-regulated in response to extrinsic stimulation with GDNF (glial cell line-derived neurotrophic factor). In cross-sections of prepubertal and adult testes, biochemicalanalyses localized POU3F1 to spermatogonia. A reduction in Pou3f1 expression induced apoptosis of cultured germ cells [72].

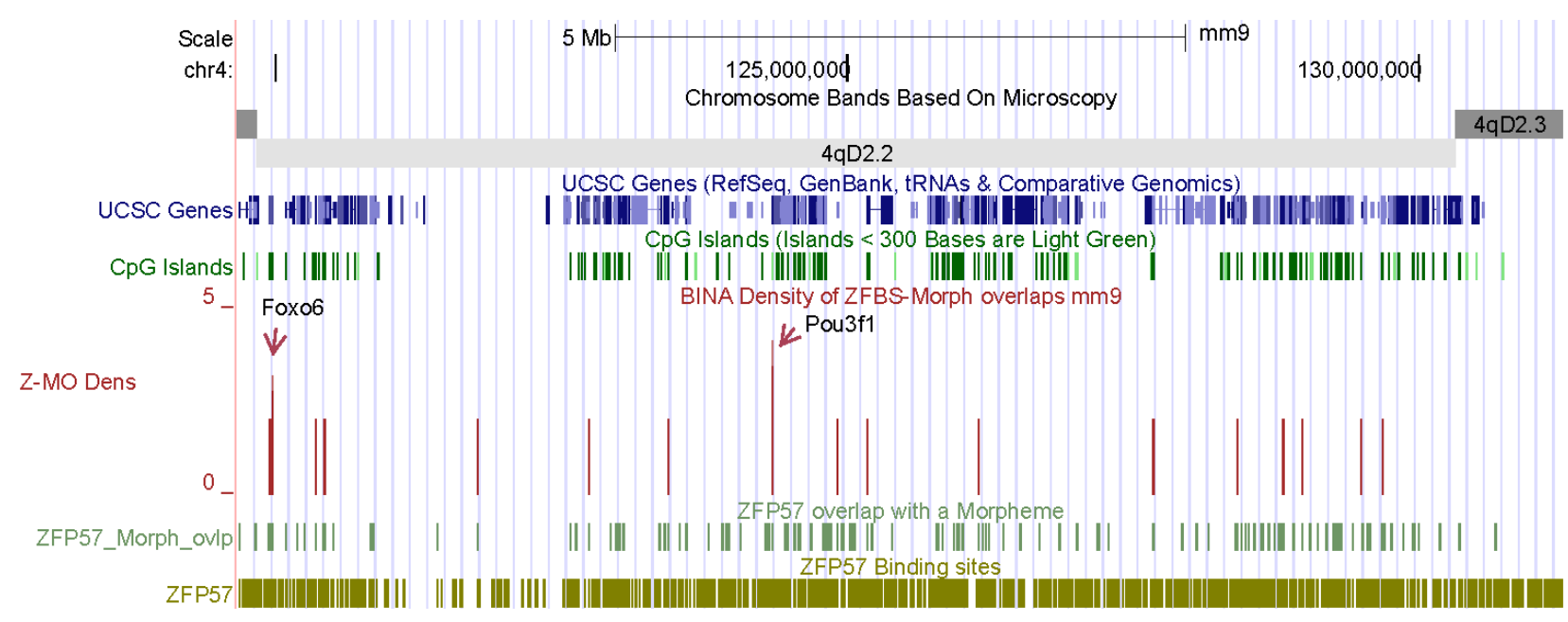

Figure 12. Two candidate ICRs mapping to two transcription factor genes (Foxo6 and Pou3f1).

In fact, in our studies we came across more than a few candidate ICRs that mapped to the vicinity of transcripts involved in sex-related processes in males and females. In previous sections we gave a few examples. Additional examples include Mgea5, Cnnm1, Chd5, and BC051142 (Figs. S24-S27). Also known as OGA, MGEA5 influences folliculogenesis [73]. Expression of Cnnm1 (cyclin M1) is associated with cell cycle and differentiation in spermatogenic cells in mouse testis [74]. Chd5 expression is restricted to post-meiotic spermatids [75]. CHD5 (chromodomain helicase DNA binding protein 5) mediates histone-to-protamine replacement impacting the cascade of molecular events underlying chromatin remodeling during spermatogenesis [75]. Chd5 expression peaks just as the most dramatic chromatin remodeling starts to take place [76]. BC051142 function is unknown. It is among the 54 evolutionarily-conserved and testis-enriched genes uncovered by genome engineering techniques [77]. Furthermore, according to GENE at NCBI, BC051142 expression is restricted toward adult mouse testes (https://www.ncbi.nlm.nih.gov/gene/?term=BC051142\#gene-expression).

\section{DISCUSSION}

To fully understand the developmental consequences of uniparental embryos, it is necessary to obtain a complete list of all imprinted genes [78]. Towards this goal, we developed a bioinformatics strategy. We built our approach on results showing that $\sim 90 \%$ of the known ICRs/gDMRs included clusters of ZFBS-morph overlaps [13, 16]. In order to remove background noise, we created plots to display the density of ZFBS-morph overlaps along genomic DNA. Previously, a study assessed the predictive potential of this strategy by examining the density-plots obtained for the human genome [19]. In this report, we assessed whether this approach also could be applied to the discovery of candidate ICRs and imprinted genes in mouse. To explore that question, initially we investigated whether we could locate known ICRs/gDMRs in relatively long DNA sections. We found that in plots, robust density peaks clearly pinpointed the essential ICR of the Gnas complex locus in $8.25 \mathrm{Mb}$ DNA (Fig. 2), the intragenic ICR of Mest in $36 \mathrm{Mb}$ DNA (Fig. 3), the ICR of Zac1 in 34 Mb DNA (Fig. 4), the ICR of the Igf2r - Airn 
imprinted domain in $23 \mathrm{Mb}$ DNA (Fig. 5), and the ICR of Nnat in 12.6 Mb DNA (Fig. 6). Based on the above and related observations, we deduced that in the density-plots, robust peaks reflected the position of actual or candidate ICRs. We posited that the genes in the vicinity of candidate ICRs corresponded to potential imprinted genes. To explore these ideas, we sampled several of the robust peaks to inspect their positions with respect to genomic landmarks, including genes, transcripts, and the CpG islands.

1. The majority of the candidate ICRs maps to CGIs; a subset corresponds to specific gene-transcripts; a smaller subset corresponds to regions associated with proteins that produce chromatin boundaries

Many of the known ICRs coincide with CGIs [5]. Similarly, we found that in the density-plots, the analyzed robust peaks primarily mapped to CGls. These islands are dispersed in various genomic locations including $1^{\text {st }}$ exons, near TSSs, and within intra and intergenic sequences. In many cases, a known ICR/gDMR represses the expression of one transcript or a group of transcripts $[60,79,80]$. Similarly, we found candidate ICRs that mapped to a single of a subset of gene-transcripts. Examples for a single transcript includes Arid1b, Nkx2-4, Six1, and Pou3f1 (Figs. 7, S15, S16 and S21). Examples for a subset of transcripts include the predicted ICRs that map to Bcl211, Taf4a, Lsm14b, Impdh1, Zbtb2, and Nfix loci (Figs. 10, S1, S2, S6, S18). In a few cases, a known ICR/gDMR is intergenic. Similarly, we found candidate ICRs within intergenic sequences (i.e., Figs. S4 and S7). Also, in a few instances, a known ICR/gDMR contains sites for binding CTCF to produce chromatin boundaries $[20,21]$. Through association with CTCF, Rad21 and SMC3 contribute to the establishment or maintenance of topological domains [81]. In our analyses we found a few candidate ICRs that mapped to or near chromatin boundaries consisting of CTCF, with or without Rad21 and SMC3. Examples include Bc/2/1, Impdh1, Cited2, Six1, and Hectd1 (Figs. 10, S2, S9, S16, and S17).

\section{Several of the candidate ICRs map to genes with functions ranging from developmental to sex-related processes}

Many of the imprinted genes are involved in regulation of embryonic growth and developmental processes. Others play key roles in neurological processes and behavior [82]. Therefore, for our studies we conducted literature surveys to investigate the function of the genes in the vicinity of the candidate ICRs that we examined. In the context of embryonic stem cells (ESCs), we obtained Zbtb2, Fam40b/Strip2, and Arid1b. In cells, ZBTB2 interacted with $\mathrm{CpG}$ island promoters, where it acted as a transcriptional activator [29]. STRIP2 was required for lineage commitment of murine ESCs [26]. ARID1B affected gene expression by catalyzing the removal of H3K4methyl marks from chromatin [83]. In the context of fetal growth, we obtained Hectd1. This gene encodes an enzyme required for the development of the junctional zone of the placenta. Disruption of Hectd1 resulted in mid-gestation lethality and intrauterine growth restriction [63].

In the context of developmental processes, we obtained several genes encoding transcription factors i.e., Cited2 and Foxo6. Cited2 impacts left-right patterning of the body' $\mathrm{s}$ axis [33]. Functions of FOXO6 include regulation of Hippo signaling and the growth of the craniofacial complex [71]. In the context of organogenesis, we obtained Mam/3. Even though Mam/3-null mice showed no apparent abnormalities, mice null for both Mam/1 and Mam/3 died early in the organogenic period [67]. In the context of vision, we obtained Impdh1. Mutations in the corresponding human gene caused retinitis pigmentosa -an inherited retinal degeneration characterized by the early onset of night blindness followed by a progressive loss of the visual field [24, 25]. In the context of sensory organs, we obtained Six1. This gene specifies a transcription factor that plays central roles in the pre-placodal ectoderm [62]. In the context of the brain and neuronal functions, we obtained Nfix, Pou3f3, and Hivep2. NFIX contributes to the development of the neocortex, hippocampus, and cerebellum [84]. POU3F3 influences neurogenesis, molecular identity, and the migratory destination of upper-layer cells of the cerebral cortex [68]. Hivep2 deficiency confers molecular, neuronal, and behavioral phenotypes related to schizophrenia [32].

In the context of sex-related processes, we obtained several genes. Lsm14b and Mgea5 impact oogenesis. Lsm14b encodes an RNA binding protein with an essential role in oocyte meiotic maturation [23]. 
Also known as OGA, MGEA5 catalyzes the removal of $\mathrm{N}$-acetylglucosamine from serine and threonine residues in many proteins [85]. Transcriptome profiling identified this enzyme as an upstream regulator of granulosa cells of bovine ovarian follicles [73]. For spermatogenesis, we obtained Pou3f1, Cnnm1, Chd5, an uncharacterized gene (BC051142), and tctex-1 locus. POU3F1 is involved in survival and self-renewal of mouse spermatogonial stem cells [72]. CNNM1 is produced in mouse testes from neonatal to adult stages [74]. CHD5 mediates histoneto-protamine replacement and impacts the cascade of molecular events underlying chromatin remodeling during spermatogenesis [75]. Among sex-related loci, the most intriguing could be the tctex-1 locus within the proximal half of Chr17 [48]. The t-haplotypes include this chromosomal section -They are variant alleles of genes linked together by four inversions $[46,47]$. While females carrying two t-haplotypes are fertile, males are sterile. The spermatozoa in males exhibit motility defects, rendering them unable to penetrate zona pellucida-free oocytes $[46,47]$. Dynlt1e is among the genes in tctex-1 -a candidate gene family for a mouse t complex sterility [48]. Our data indicate that in the proximal half of chromosome 17, the tctex-1 locus might be regulated by genomic imprinting through a candidate ICR that maps to a CGI upstream of one of the Dynlt1e genes/transcripts. Consistent with this interpretation are several H3K9me3 marks dispersed across the entire tctex-1 locus (Fig. 8).

\section{CONCLUSION}

This report offered a strategy for simultaneous discovery of candidate ICRs and imprinted genes in the mouse genome. With this approach, we could clearly discern the genomic positions of several of the known ICRs/gDMRs within relatively long DNA sections. Furthermore, with respect to predicted ICRs, we could locate potential imprinted genes/transcripts with diverse and physiologically important functions -including fetal grow, organogenesis, craniofacial feature, patterning of the body axis, development of sensory organs, several sex related processes, and chromatin remodeling. These and related findings could lend further support for robustness of our strategy. Nonetheless, only experimental validations could demonstrate the strength of our approach. Therefore, we offer links for accessing and downloading our data on the positions of ZFBS and ZFBSMorph overlaps [86], peaks in the density-plots [87], and the MLL1 morphemes in the build mm9 of the mouse genome [88]. Also available are links for downloading data-files obtained for the build hg19 of the human genome [89-91].

\section{METHODS}

Previously, two reports gave links for accessing the genomic positions of a set of composite DNA elements consisting of the ZFP57 binding site overlapping a subset of the MLL1 morphemes $[13,86]$. We wrote a Perl script for creating the density-plots. The script opened the file containing the positions of ZFBS-Morph overlaps in a specified chromosome. Next, the script scanned the file to count and to report the number of ZFBS-Morph overlaps within a sliding window consisting of 850 bases. By omitting isolated occurrences, the script removed background noise. Subsequently, we combined and tailored the outputs of the program for upload as a custom track at the UCSC genome browser. Several publications offer concise overviews about how to use the UCSC browser [38, 39]. Default tracks at the browser include the positions of genes, transcripts, the CpG islands, SNPs, and the ENCODE data [37,61]. The positions of SNPs could facilitate designing primers to test the validity of our predictions. With the ENCODE data, one could examine the candidate ICRs in the context of histone marks, DNase I hypersensitive sites, and results of ChIPs determined for several nuclear proteins including CTCF, RAD21, and SMC3; for details, see reference [92].

\section{Competing interests: none}

\section{Acknowledgments}

We thank Zena Narod Lamp for editing our manuscript, Arnold Stein for helpful discussions. 


\section{SUPPLEMENTAL FIGURES}

Fig. S1. A close-up view of the Taf4a and Lsm14b loci. A candidate ICR maps to the longest Taf4a transcript, another one maps to the longest $L s m 14 b$ transcript. A short DNA segment includes the TSSs of both Taf4a and 4921531C22Rik (a testis-specific gene).

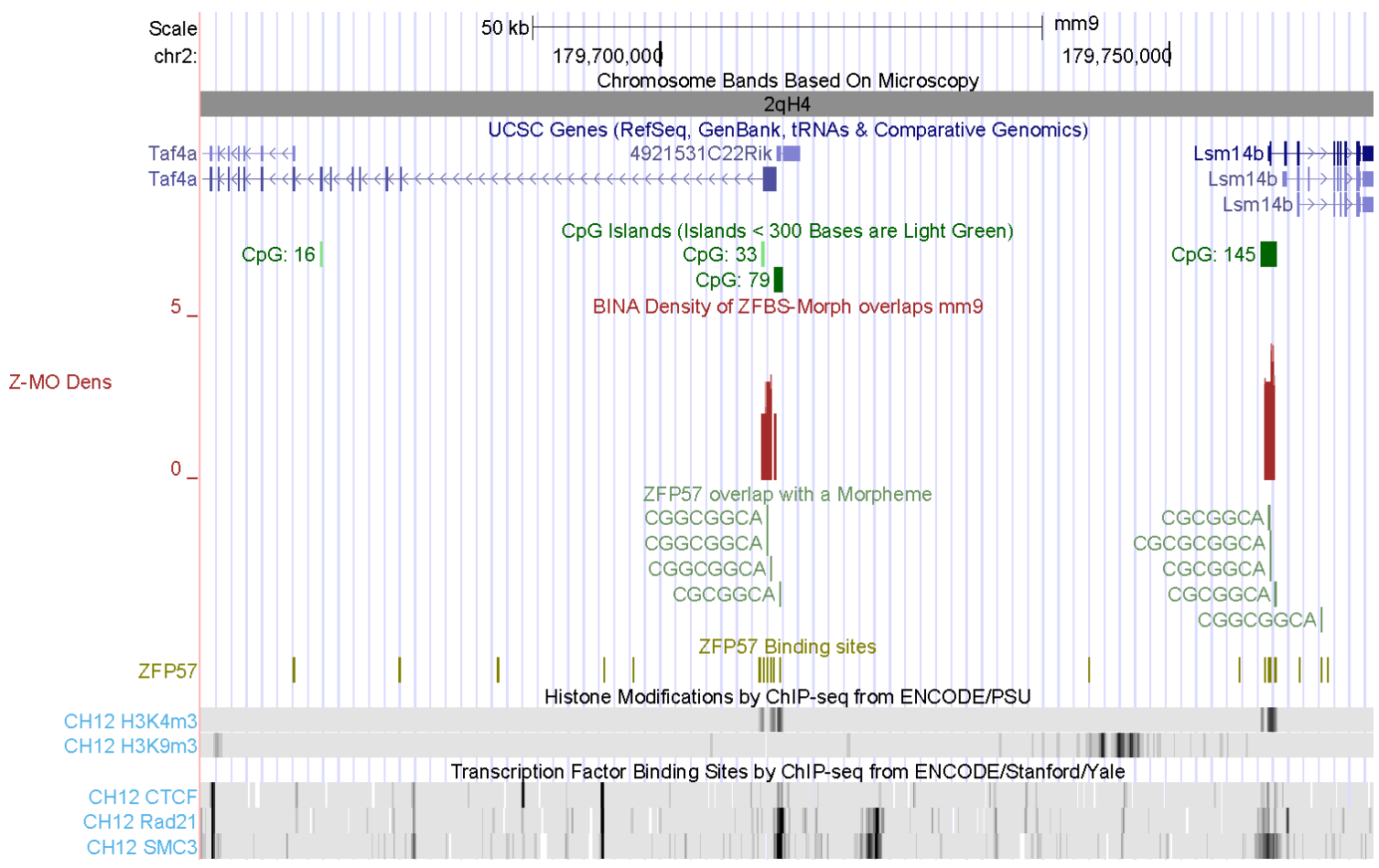

Fig. S2. The position of a candidate ICR with respect to the longest Impdh1 transcripts. The corresponding density peak maps to a CTCF-associated region.

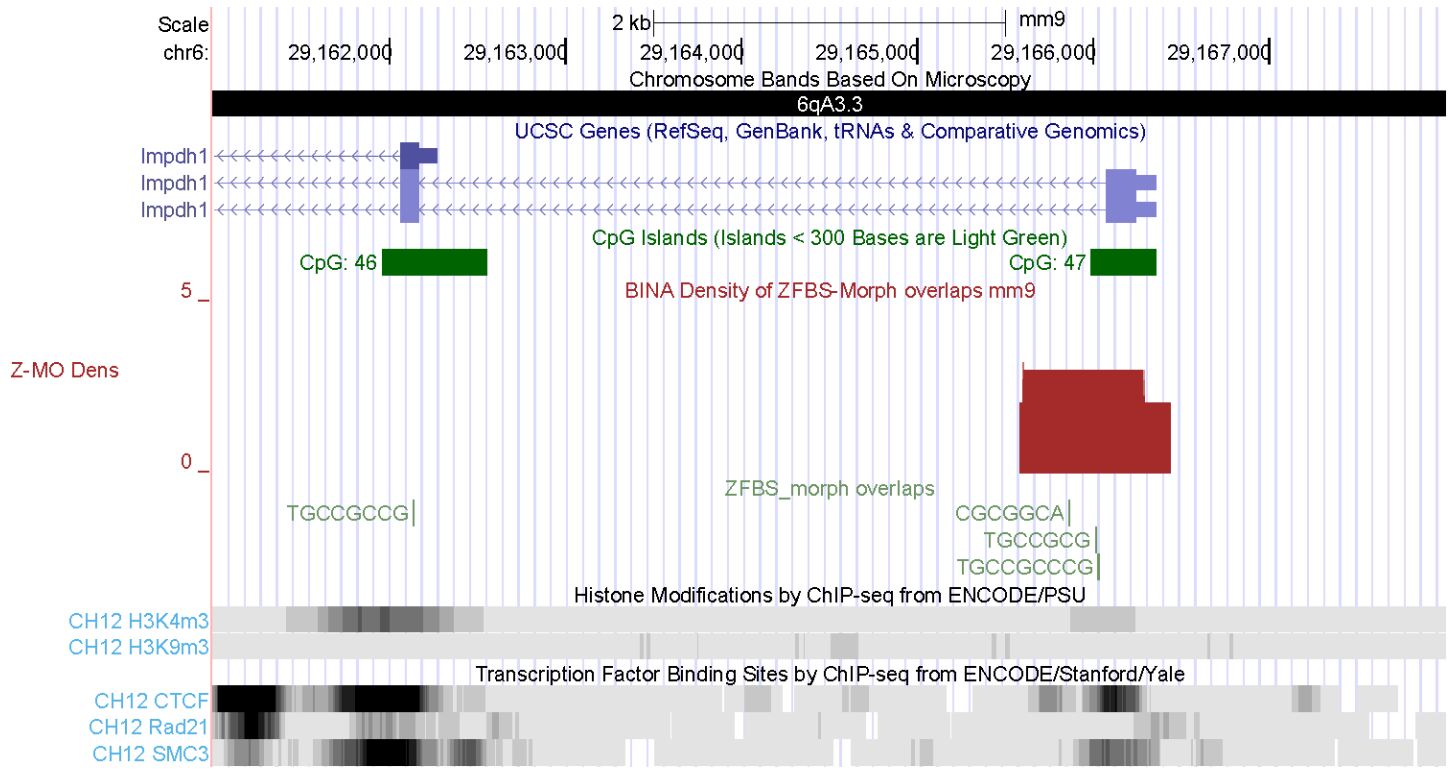


bioRxiv preprint doi: https://doi.org/10.1101/780551; this version posted September 24, 2019. The copyright holder for this preprint (which was not certified by peer review) is the author/funder. All rights reserved. No reuse allowed without permission.

Fig. S3. The position of a candidate ICR with respect to the TSS of Fam40b/ Strip2. The product of this gene plays an indispensable role in the onset of ESCs differentiation [26].

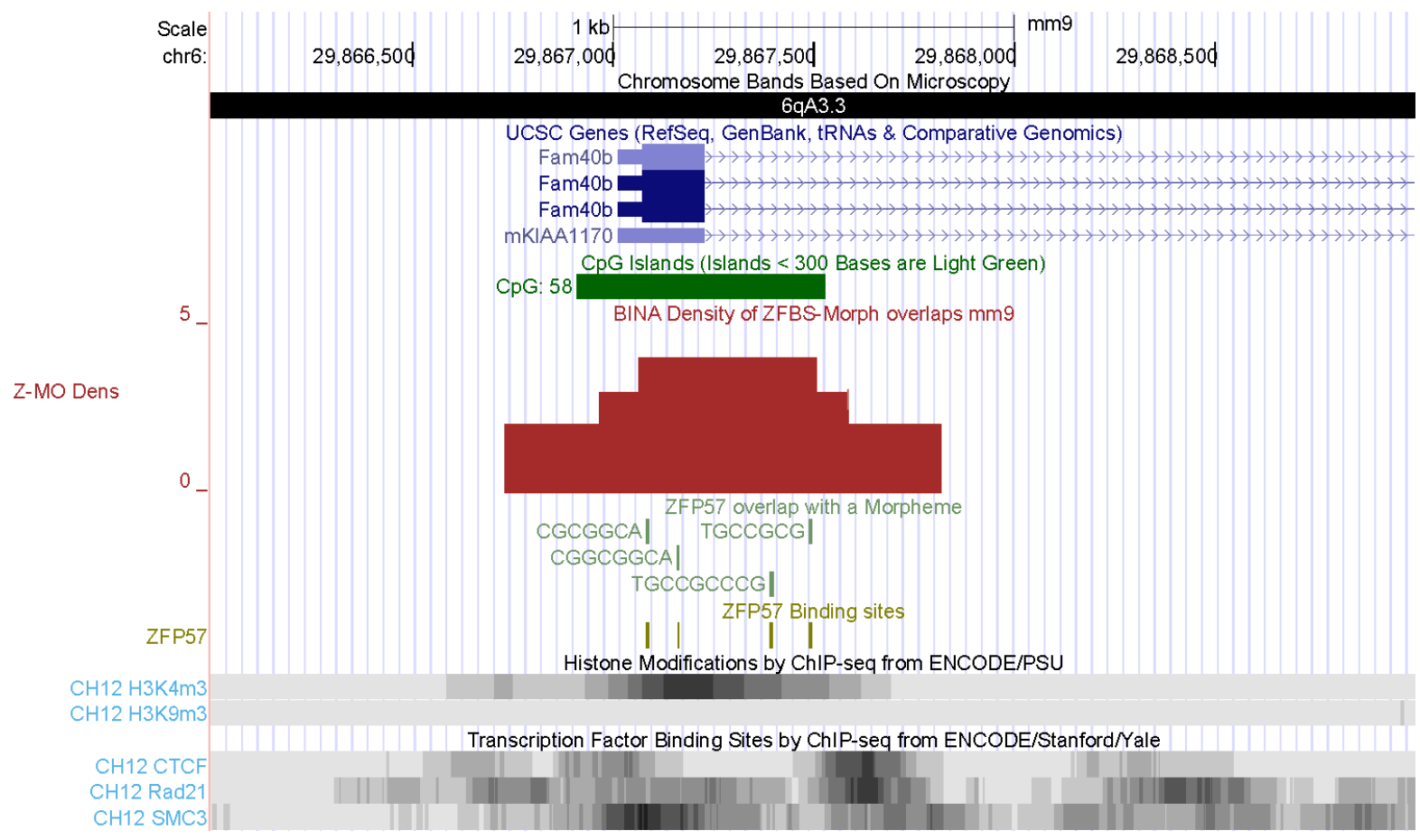

Fig. S4. The position of a candidate ICR far upstream of S/c35b4. The product of this gene regulates obesity and glucose homeostasis [27]. The displayed segment includes several dispersed H3K9me3 marks.

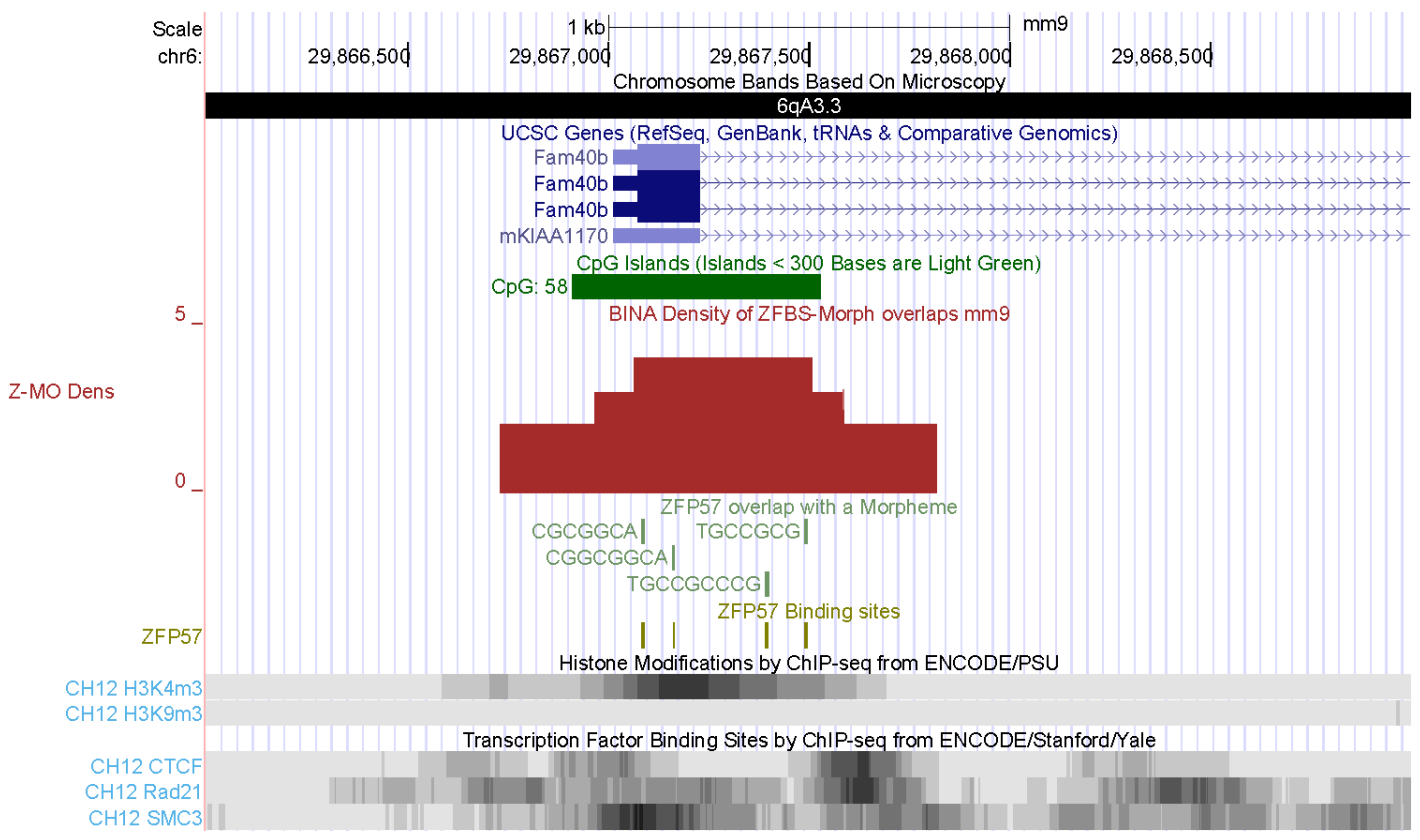


bioRxiv preprint doi: https://doi.org/10.1101/780551; this version posted September 24, 2019. The copyright holder for this preprint (which was not certified by peer review) is the author/funder. All rights reserved. No reuse allowed without permission.

Fig. S5. The position of the density peak in the Plag/1 locus. Zac1 corresponds to one of the Plag/1 transcripts. The locus includes a conserved intragenic $\mathrm{CpG}$ island that is methylated in oocytes [28]. A very robust density peak correctly located the ICR in the intragenic CpG island (CpG72). This peak covers the previously reported cluster of 5 ZFBS-Morph overlaps in the locus [16].

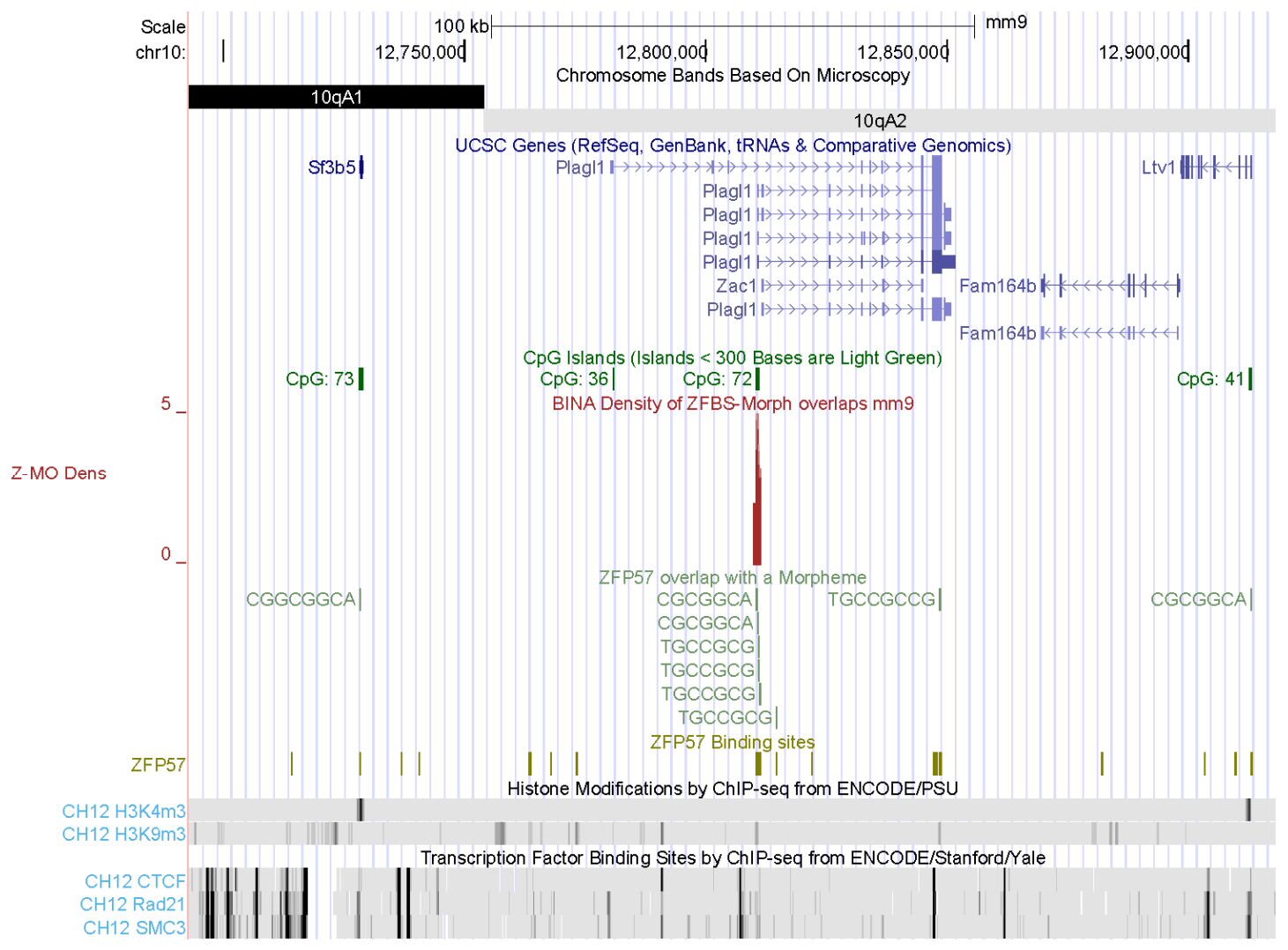

Fig. S6. The position of a candidate ICR with respect to the longest Zbtb2 transcript. Zbtb2 regulates the differentiation of mouse ES cells [29].

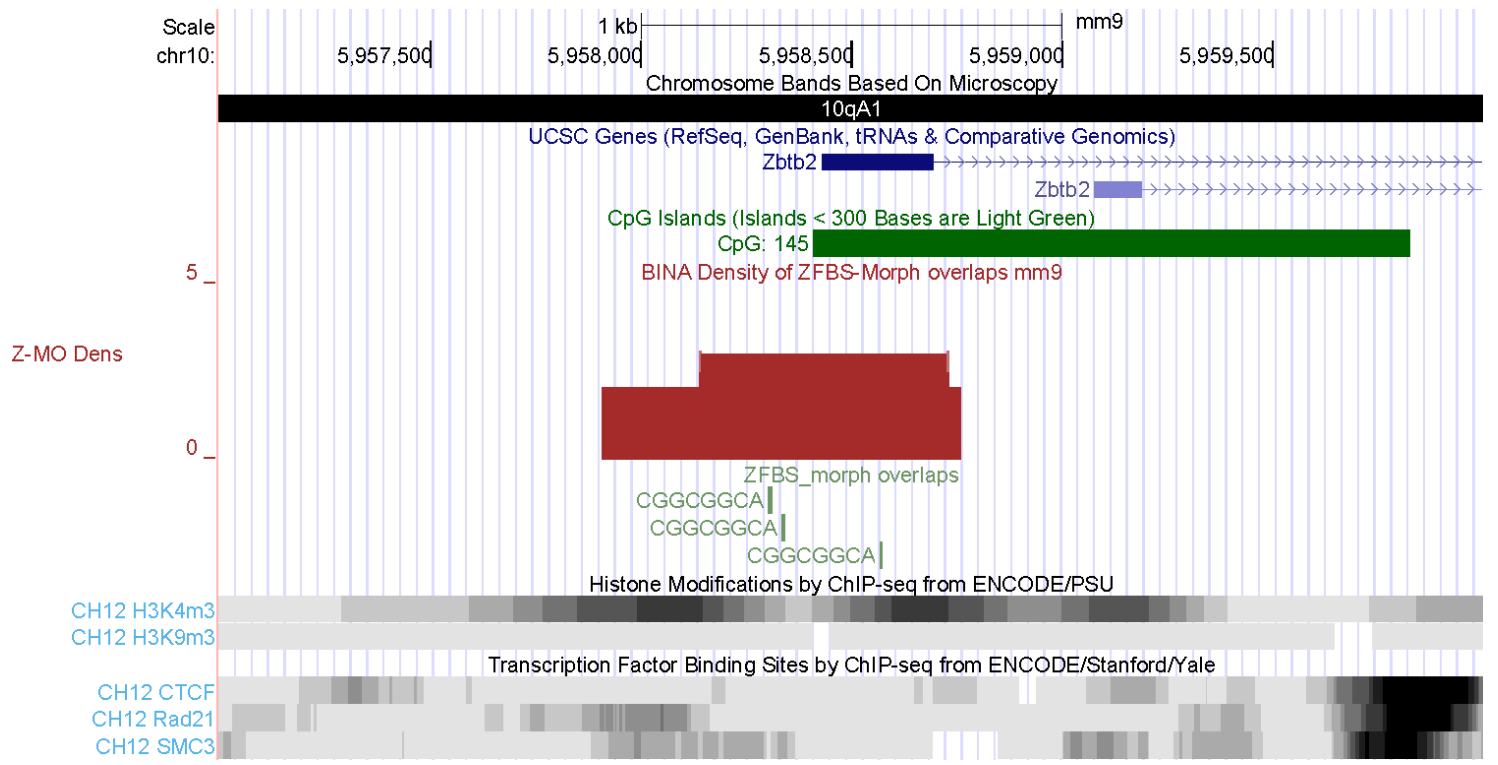


Fig. S7. The position of an intergenic candidate ICR with respect to the TSSs of Ccdc28a and Nhs/1. Ccdc28a is expressed in testes (https://www.ncbi.nlm.nih.gov/gene/215814\#gene-expression). The function of Nhs/1 is not fully understood. The displayed segment includes several dispersed H3K9me3 marks.

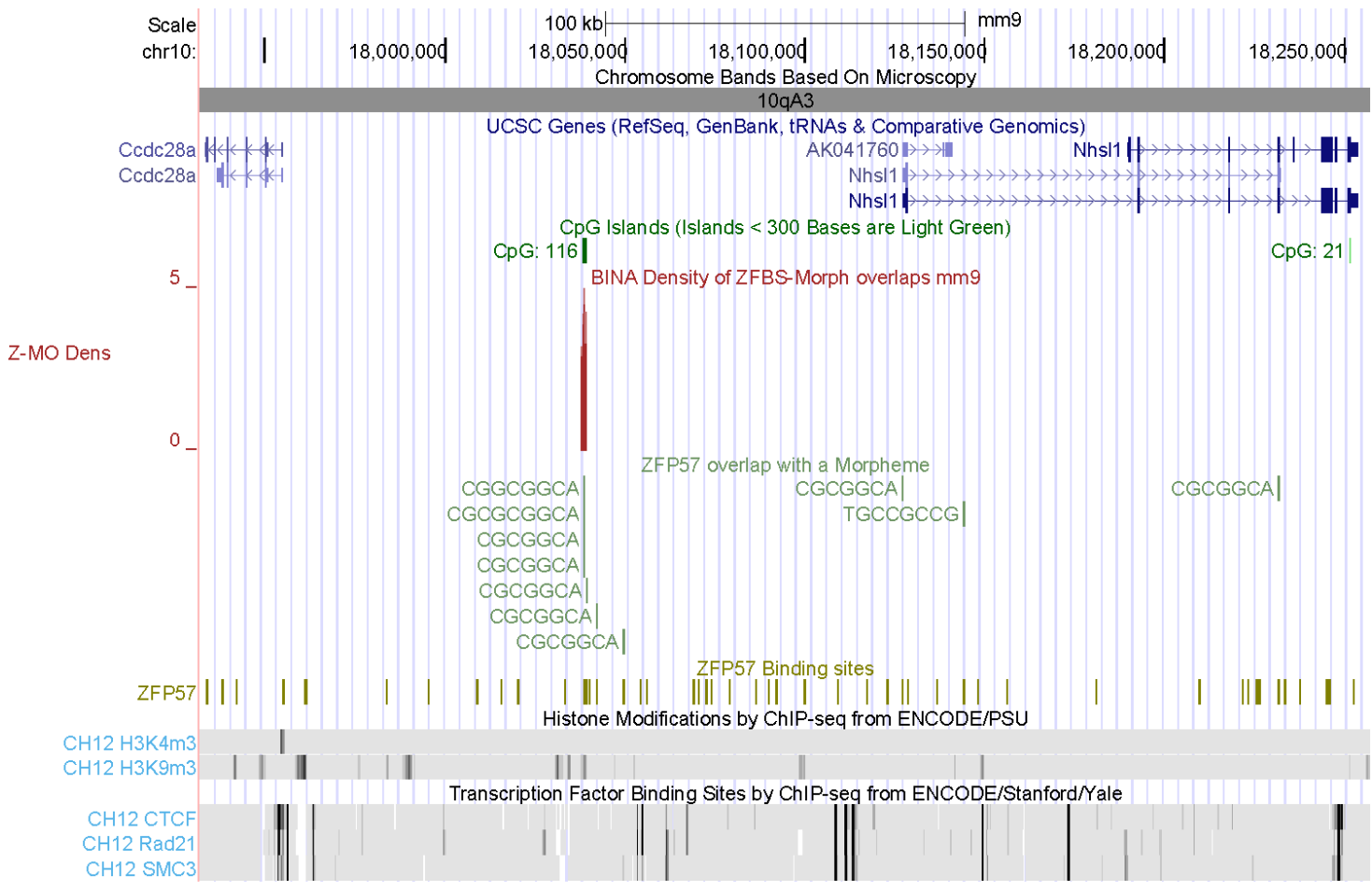

Fig. S8. The position of a candidate ICR within the Stx7 locus. The corresponding density peak overlaps a chromatin boundary consisting of CTCF, RAD21, and SMC3. Syntaxin-7 is among a group of proteins that function in vesicle transport and fusion events [30].

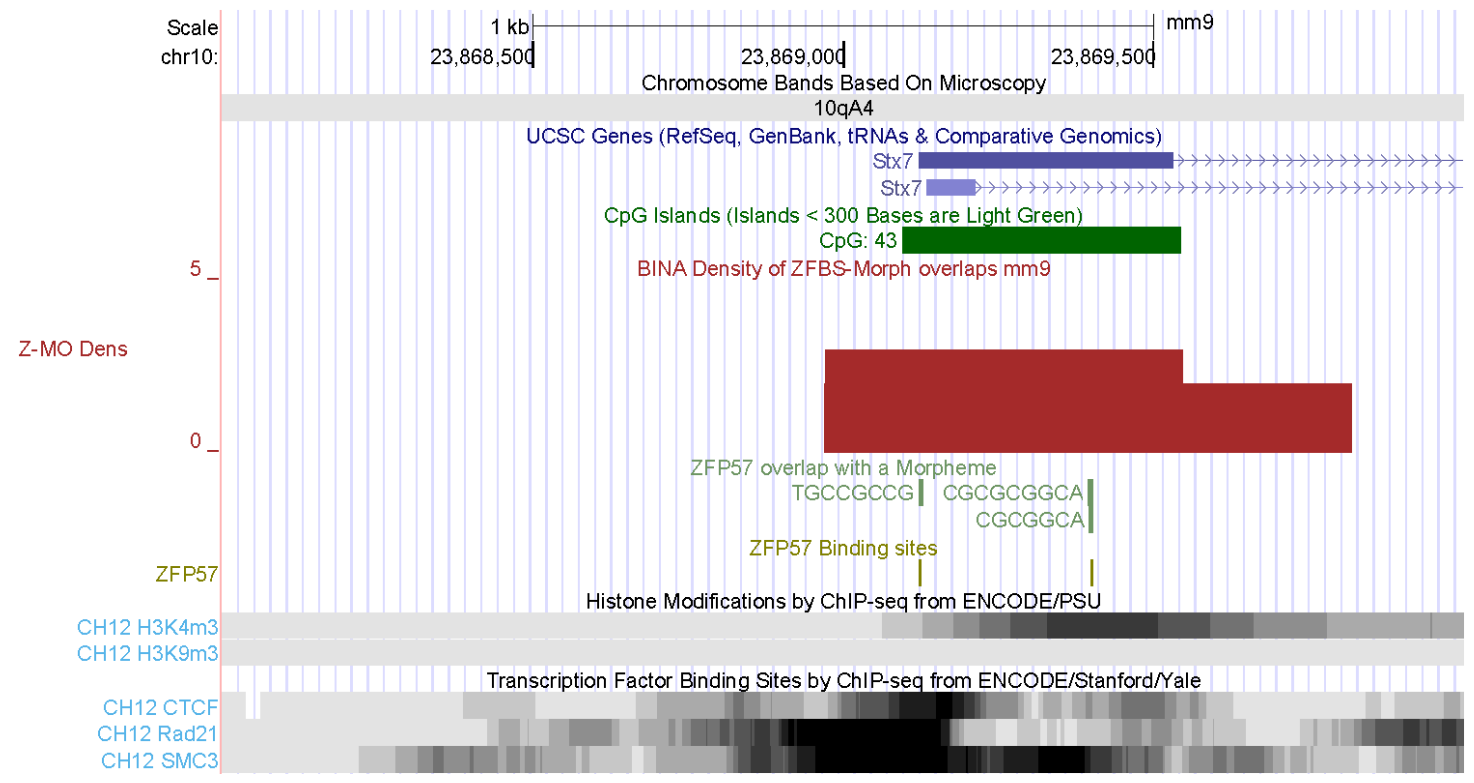


Fig. S9. The position of a candidate ICR in the Cited2 locus. This ICR contains 2 density peaks that map to chromatin boundaries. Absence of Cited 2 in mouse embryos caused congenital heart disease by perturbing leftright patterning of the body axis [33]. The locus includes several closely-spaced chromatin boundaries.

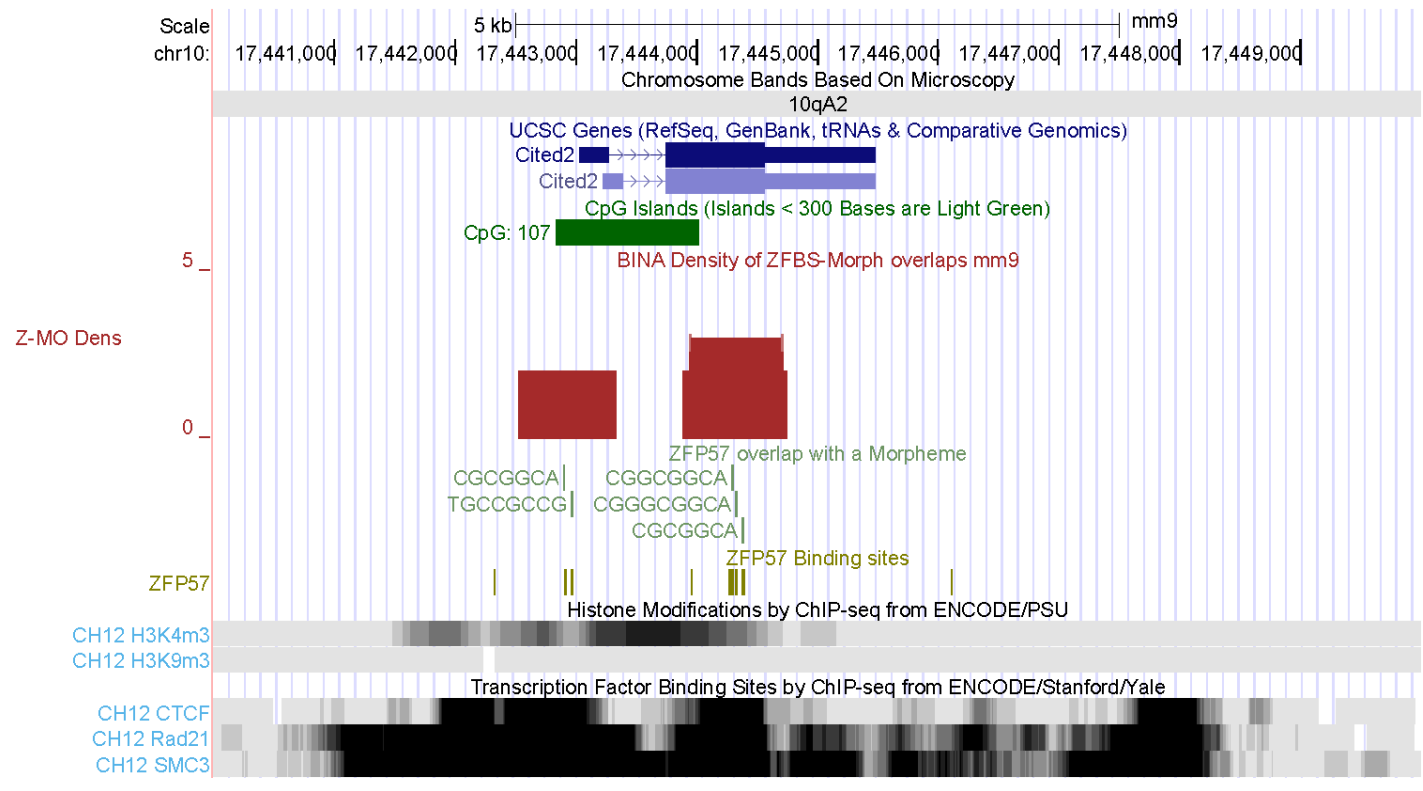

Fig. S10. An intragenic candidate ICR within the Hivep2 locus. The corresponding density peak is in a region that encompasses several chromatin boundaries. Hivep2 deficiency conferred molecular, neuronal, and behavioral phenotypes related to schizophrenia [32].

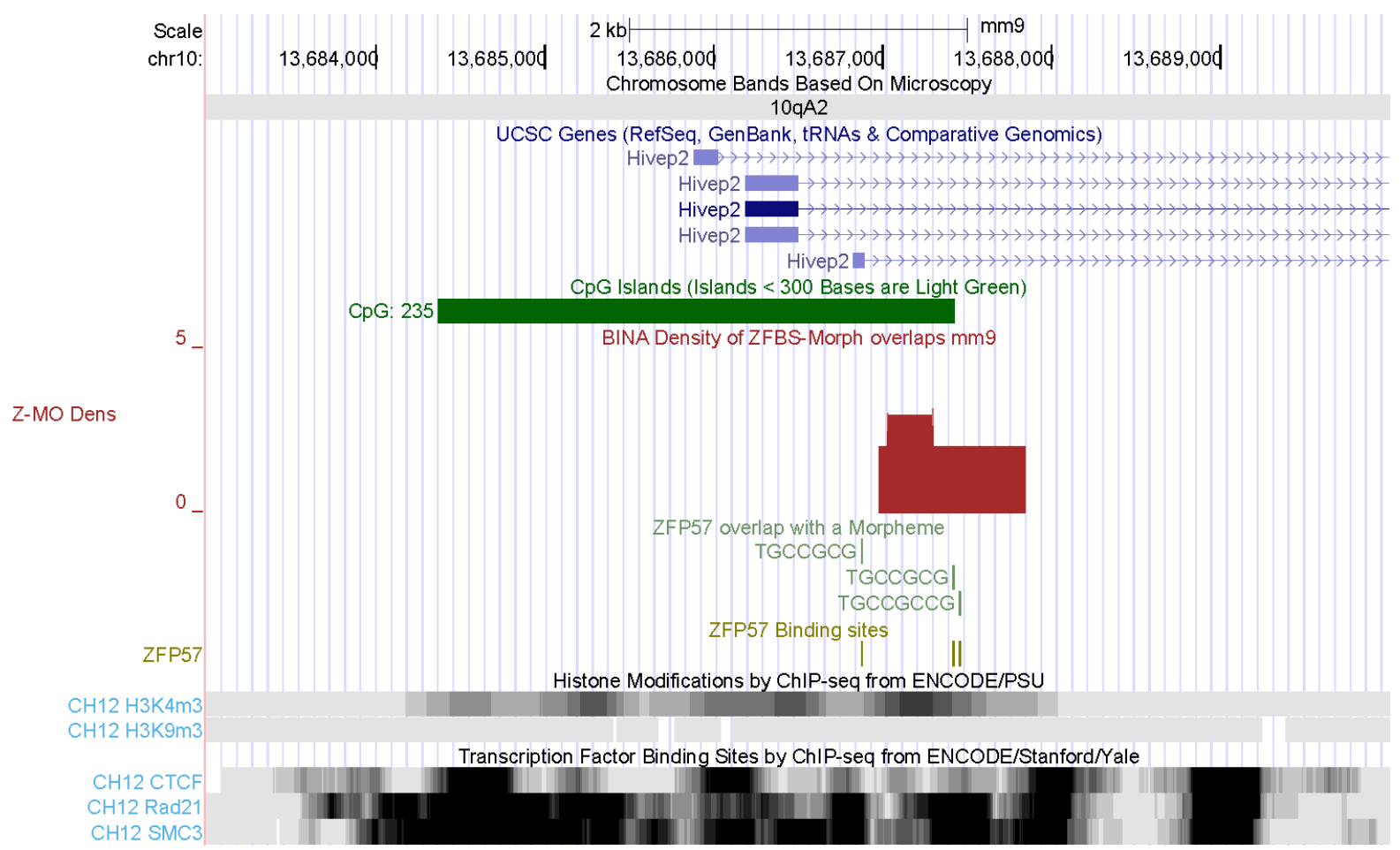


Fig. S11. The position of the density peak in the Igf2r - Airn imprinted domain. This peak covers several ZFBSmorph overlaps located in the intronic CpG island that regulates expression of genes in the imprinted domain [16]. The displayed DNA section consists of $1.17 \mathrm{Mb}$. This section also includes $T c p 1$-one of the genes carried by all $t$ haplotypes [93]. Note that there is a density peak in the longest intron of Tcp10. This gene also is in the chromosomal section that encompasses the $t$ haplotypes. Even though the peak covers only 2 ZFBS-morph overlaps, it is within a relatively long chromatin segment that includes extensive repressive H3K9me3 marks.

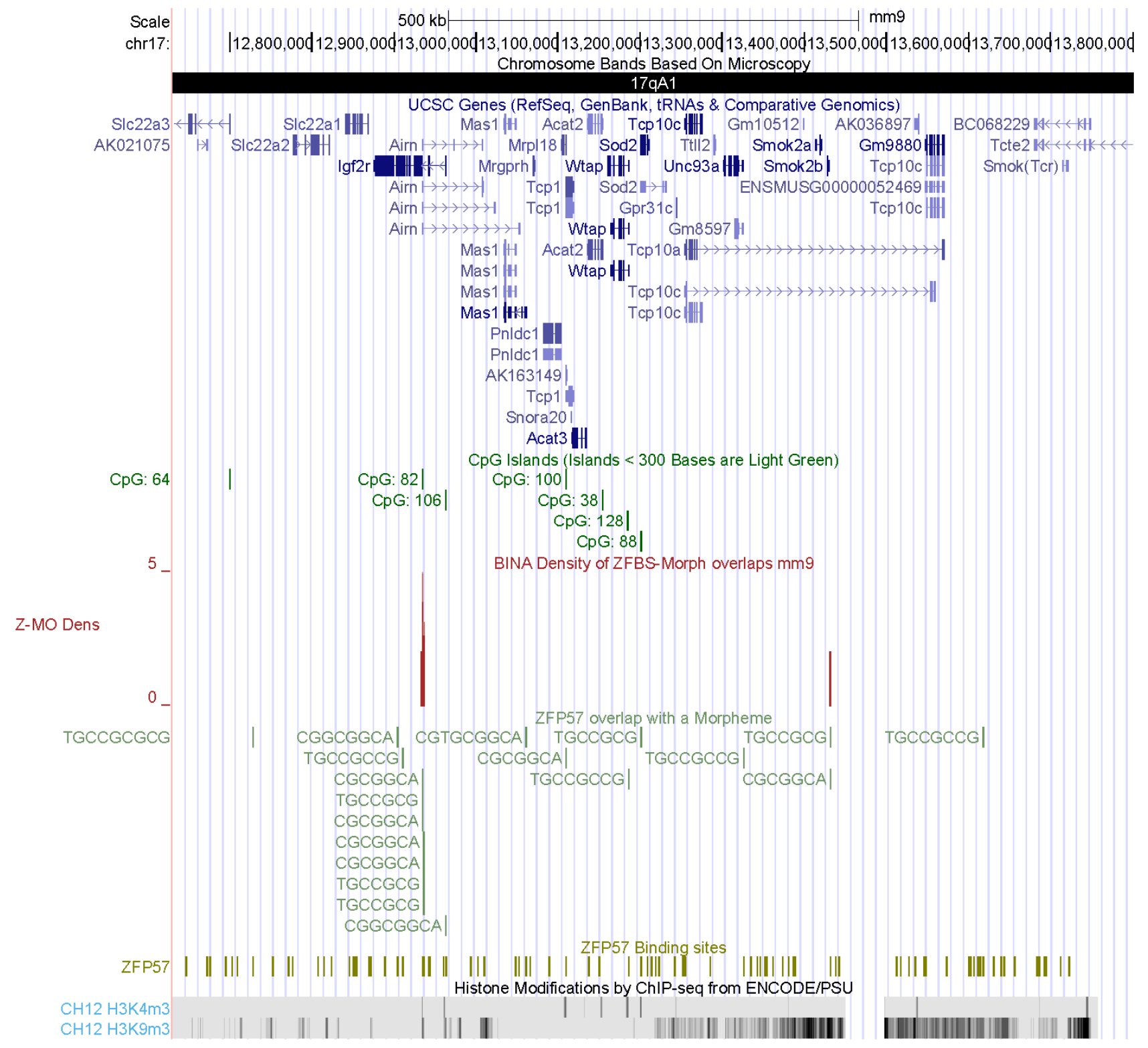


Fig. S12. The position of a candidate ICR for a gene (KRAZ1) in a chromosomal section with several ZFP genes. $K R A Z 1$ encodes a factor with Krüppel-type zinc finger structural motif [42-44]. As ZNF57, KRAZ1 interacts with KAP1/TRIM28 [43]. The displayed segment includes several dispersed repressive H3K9me3 marks.

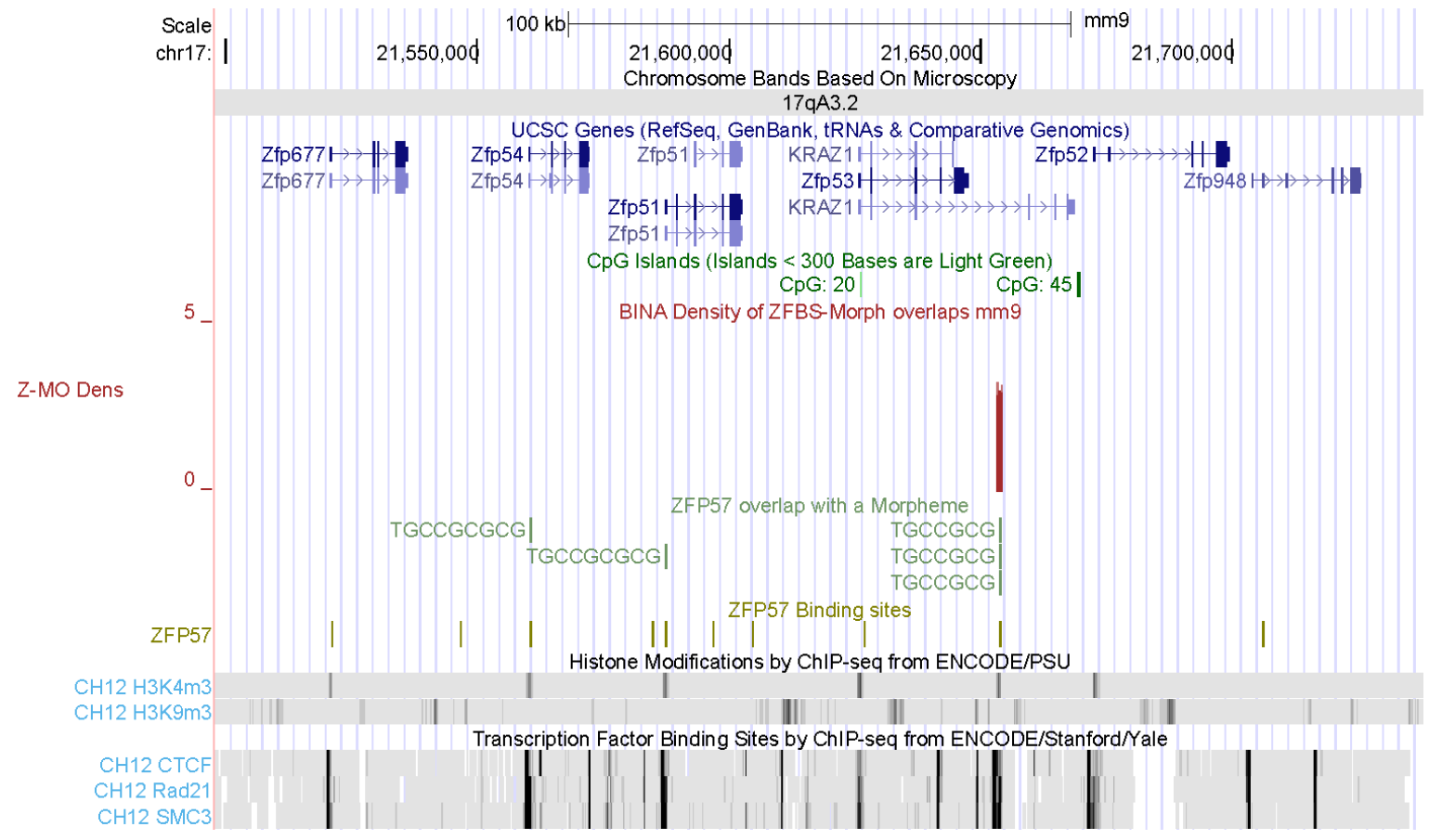

Fig.S13. Two intragenic density peaks locating the ICR of Nnat in BIcap locus.

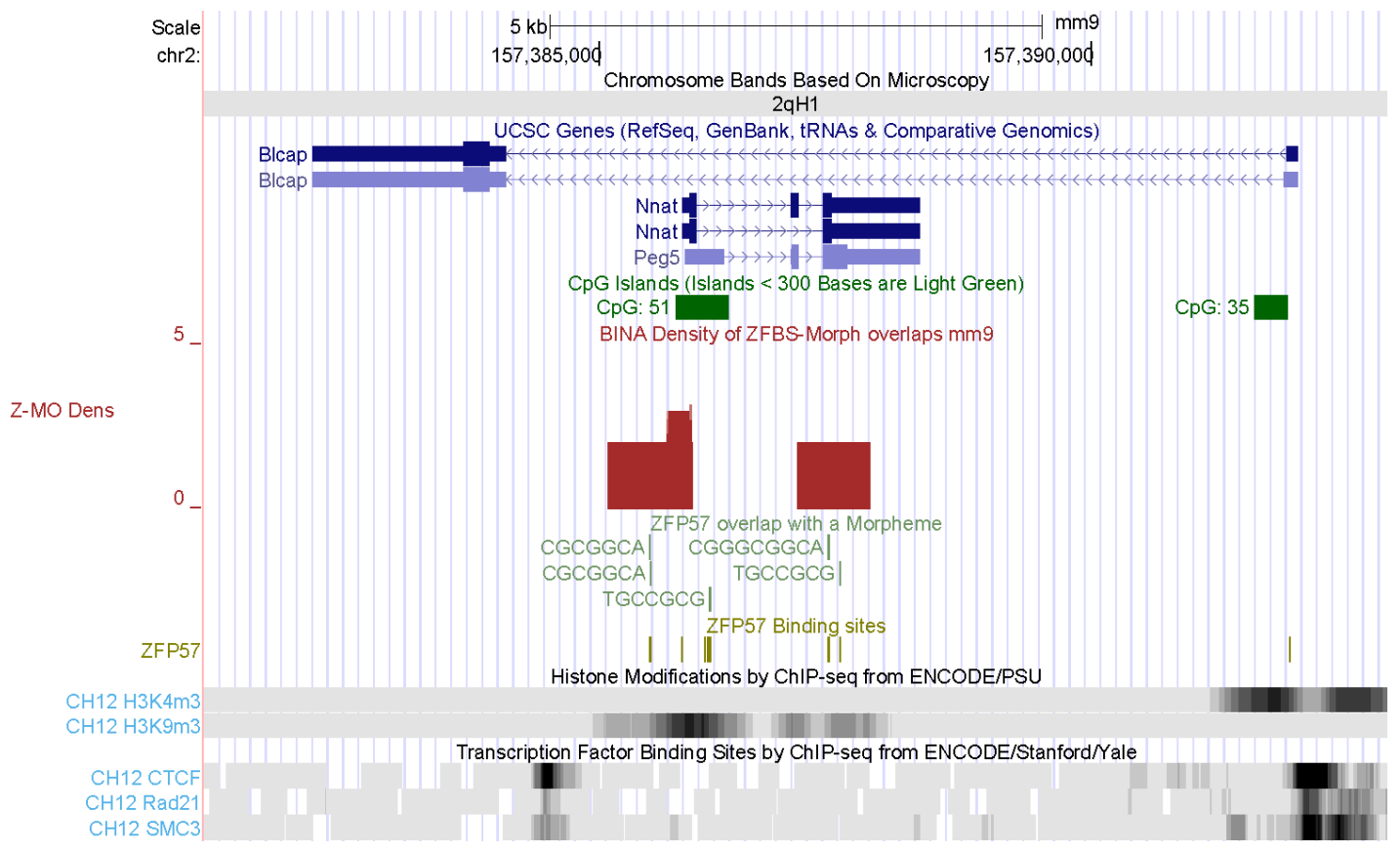


Fig. S14. A candidate ICR mapping to Ccm2/ locus. The product of this gene is selectively produced in endothelial cells during angiogenesis [57]. $\mathrm{Ccm} 2 \mathrm{r}^{-{ }^{-}}$animals exhibited embryonic lethality at E11 associated with myocardial thinning [56].

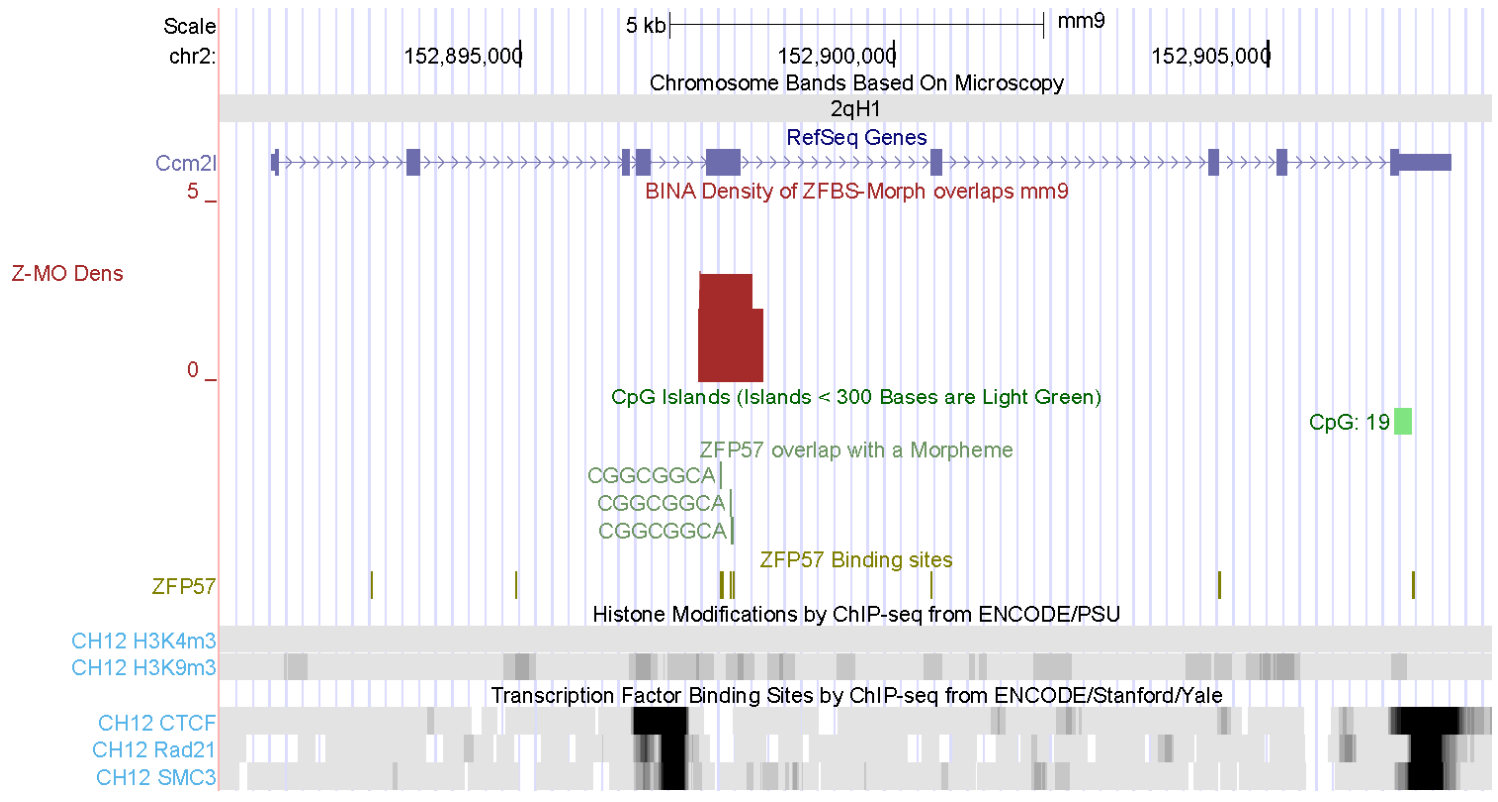

Fig. S15. The position of a candidate ICR mapping to Nkx2-4. The product of this gene is a transcription factor whose structure includes a homeodomain for binding DNA [58]. During mouse embryogenesis, Nkx-2.2 transcripts were dispersed in localized domains of the brain and might be involved in specifying diencephalic neuromeric boundaries [58]. The displayed segment includes dispersed H3K9m3 marks.

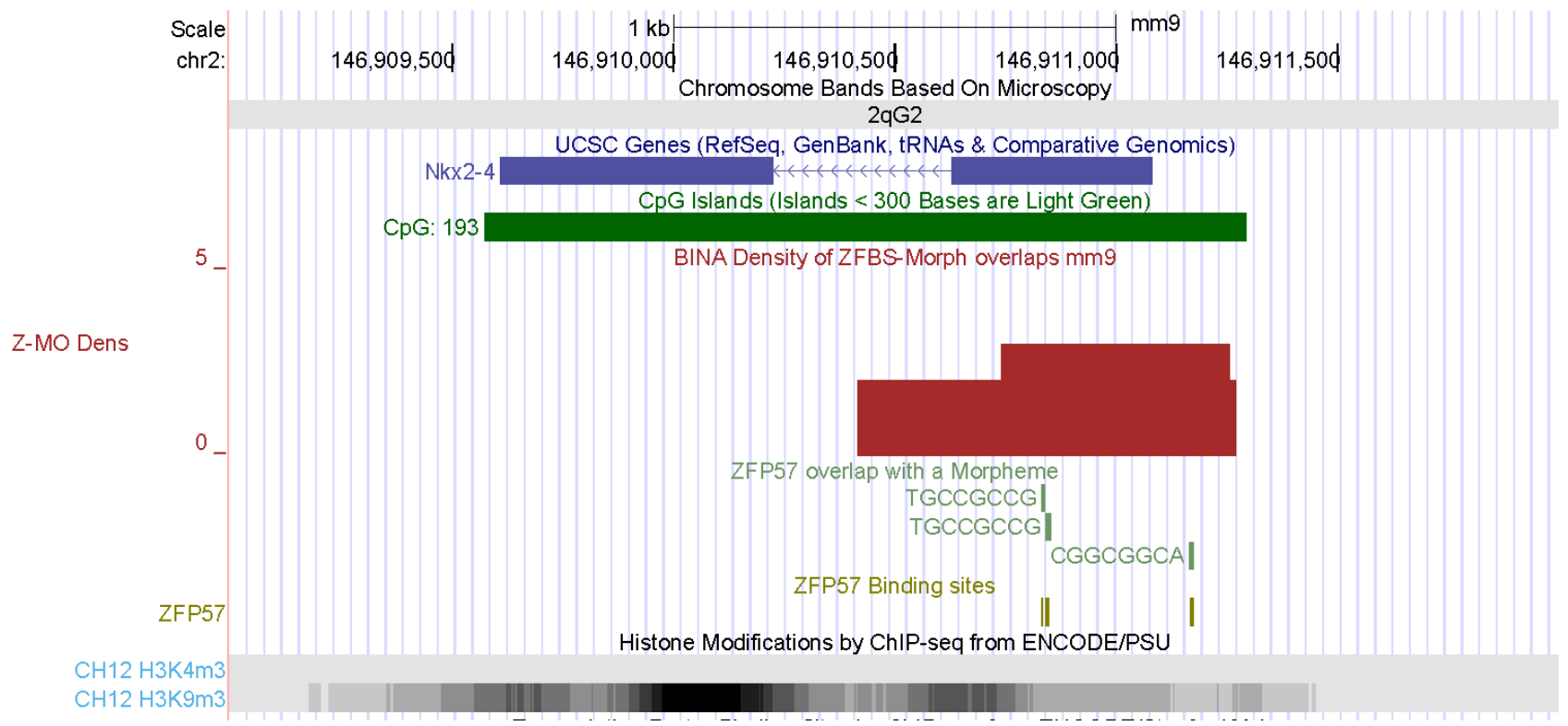


Fig. S16. A candidate ICR in a DNA segment with several Six genes. The corresponding density peak maps to Six1. This gene specifies a transcription factor (SIX1) that is a component of a protein-network that controls organ development [62]. Six1-deficient mice embryos were devoid of inner ear structures [94].

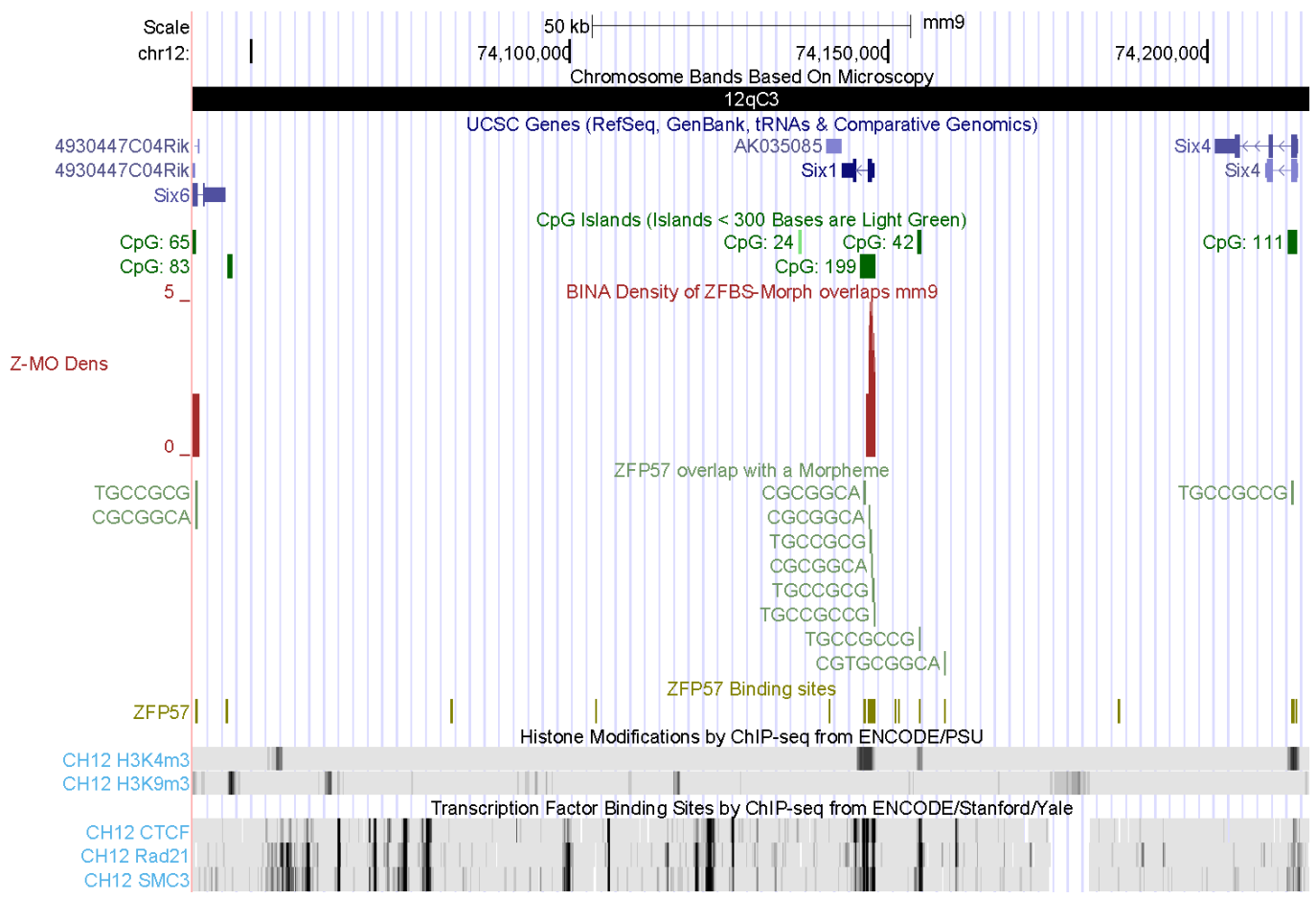

Fig. S17. The position of a candidate ICR mapping to Hectd1 locus. The corresponding density peak is within chromatin boundaries. Disruption of Hectd1 resulted in mid-gestation lethality and intrauterine growth restriction [63].

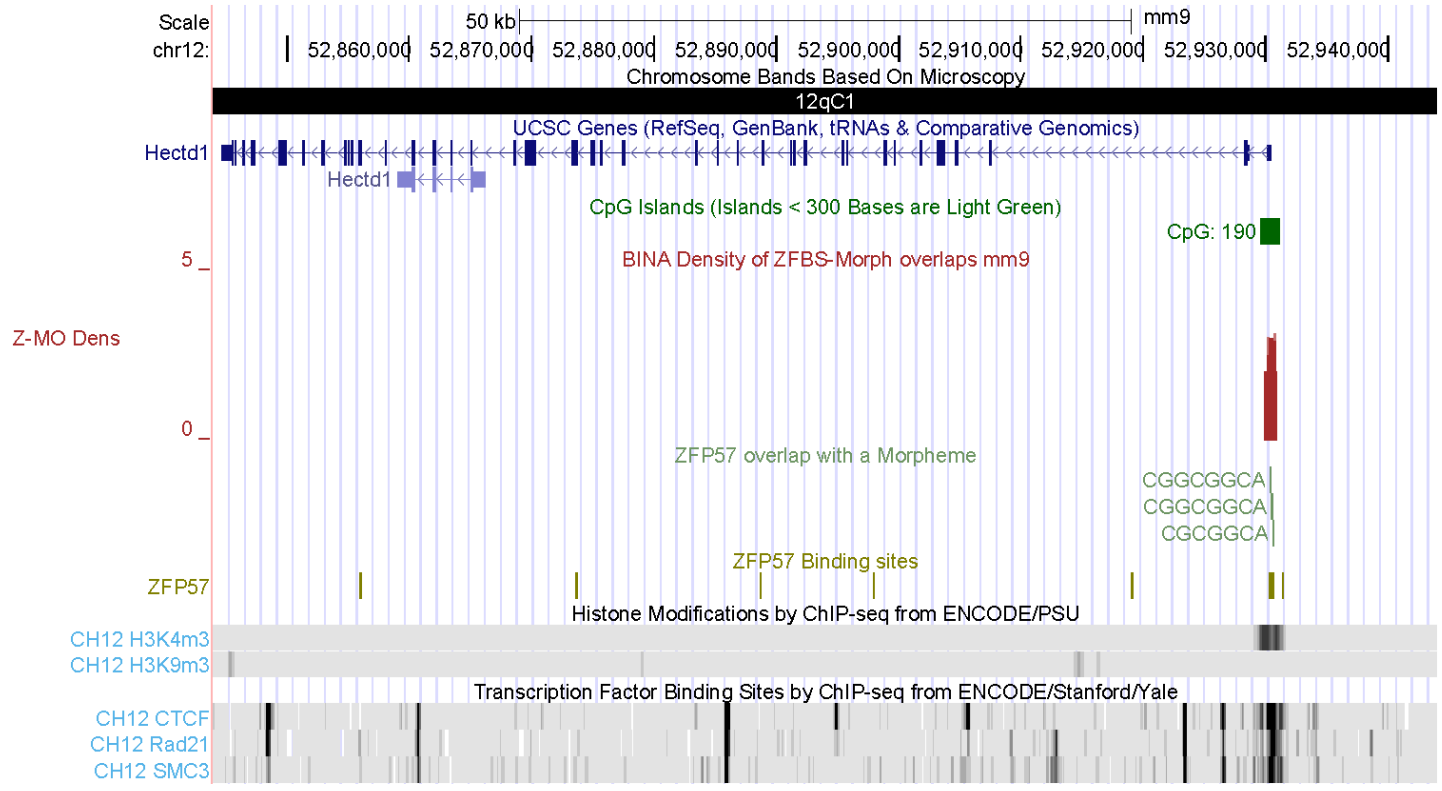


Fig. S18. The position of a candidate ICR mapping to the longest Nfix transcripts. NFIX belongs to a family of transcription factors that play important roles in the development of the nervous system [66]. The locus includes dispersed $\mathrm{H} 3 \mathrm{~K} 9 \mathrm{me} 3$ marks.

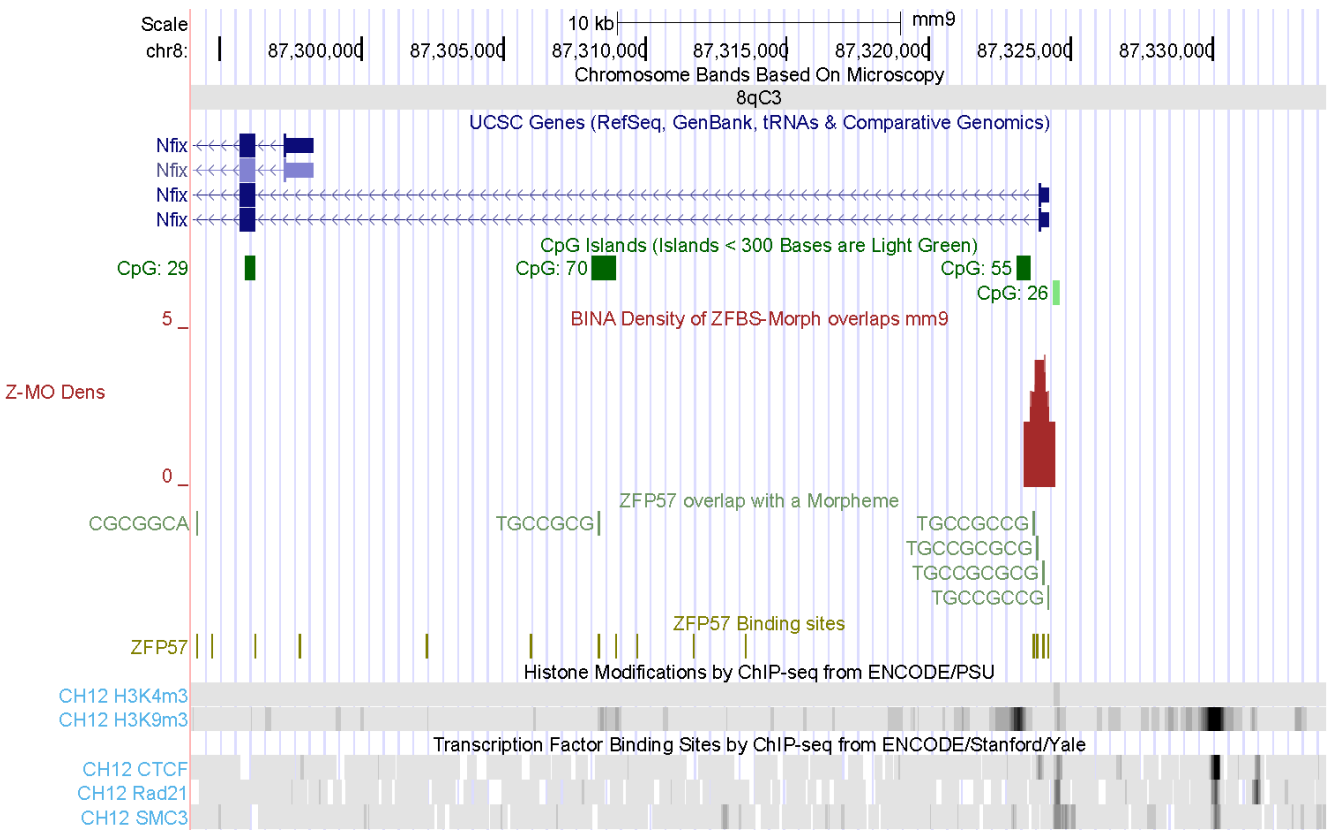

Fig. S19. The position of a candidate ICR mapping to the longest of Mam/3 transcript. This candidate ICR is far upstream of Foxo1 TSS. Mam/3 functions in organogenesis [67]. Across mammals, Foxo1 functions in both male and female reproductive processes [95].

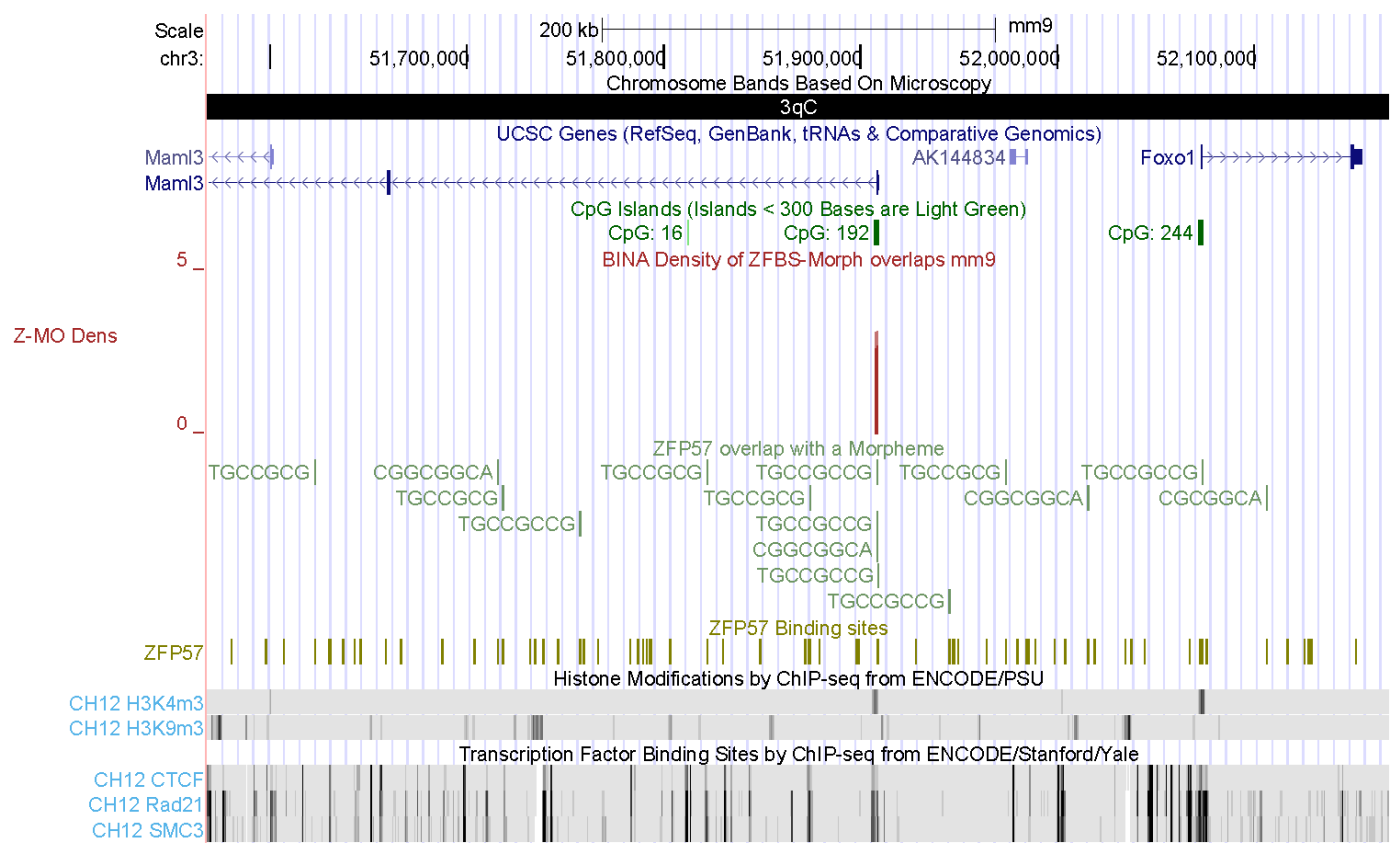


Fig. S20. Density peaks defining a candidate ICR for Pou3f3. POU3F3 influences neurogenesis [68]. Upstream of Pou3f3 is the TSS of a noncoding RNA gene (Pantr1). This RNA is differentially expressed during neural stem cell differentiation [96]. Dispersed between Pou3f3 and Pantr1 are several regions containing repressive H3K9m3 marks.

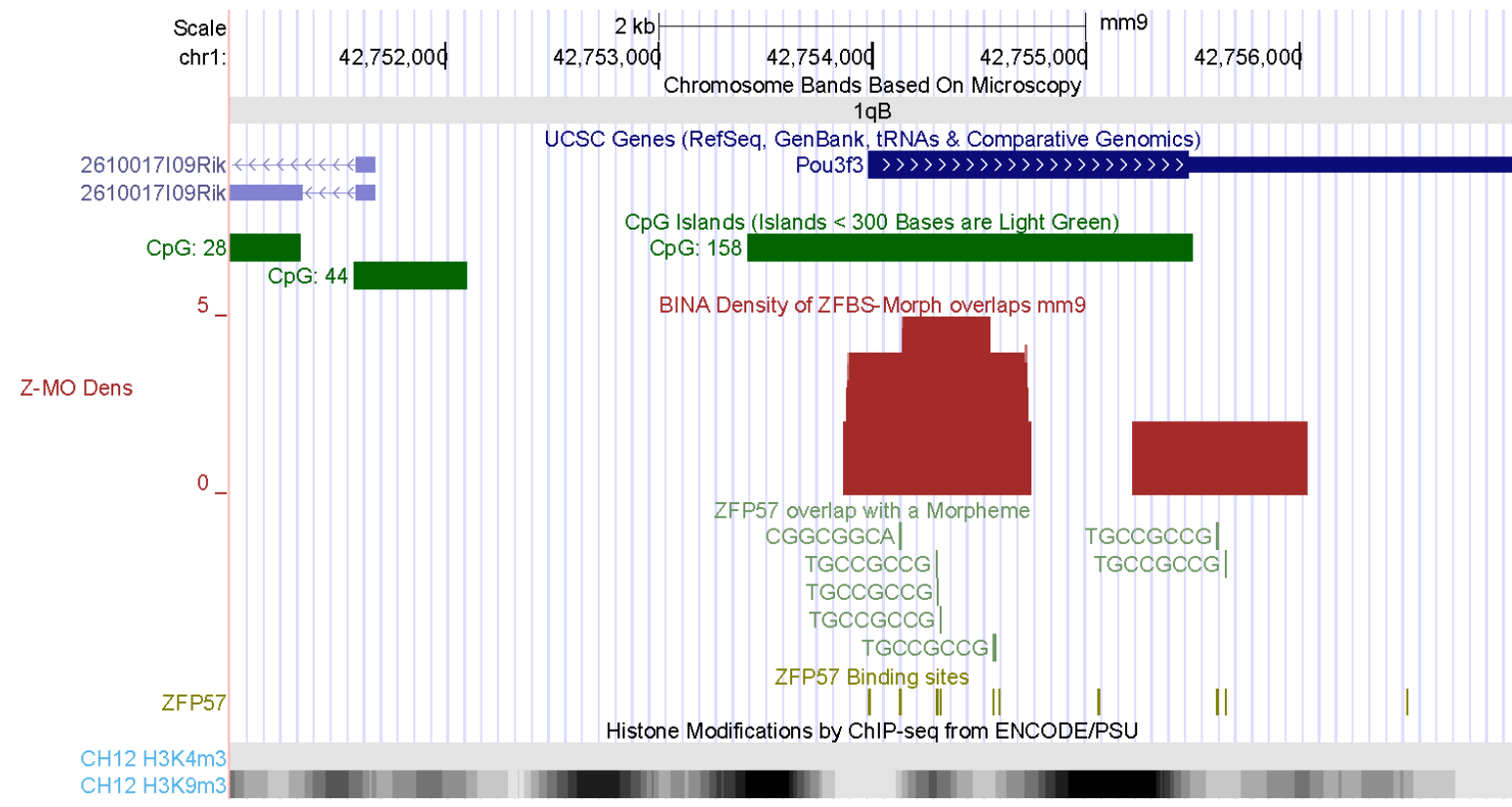

Fig.S21. The position of a candidate ICR mapping to Rev1 locus. This gene encodes a DNA polymerase that during somatic hypermutation incorporates deoxycytidine residues most likely opposite abasic nucleotides [97].

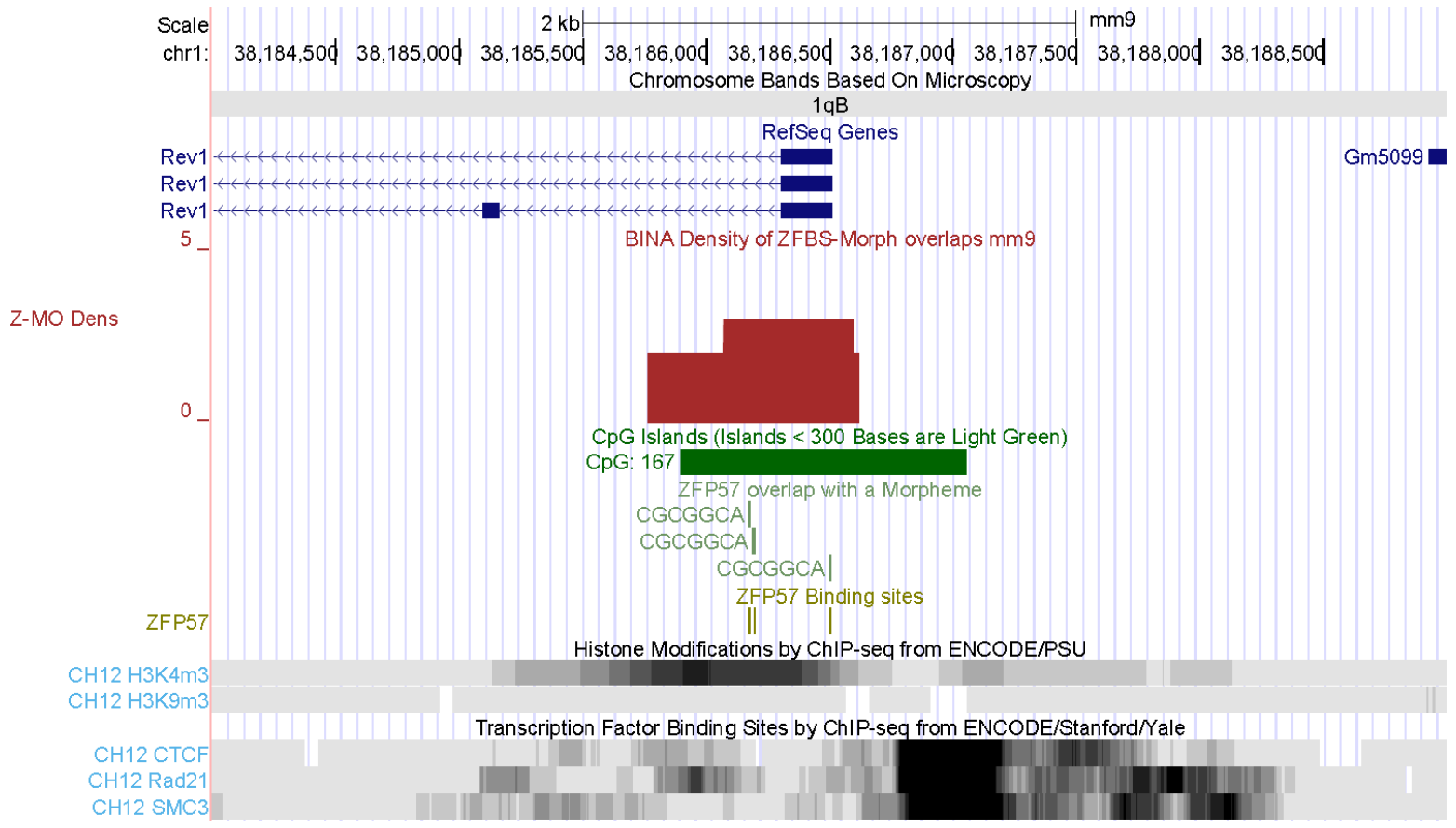


bioRxiv preprint doi: https://doi.org/10.1101/780551; this version posted September 24, 2019. The copyright holder for this preprint (which was not certified by peer review) is the author/funder. All rights reserved. No reuse allowed without permission.

Fig. S22. The position of a candidate ICR for Foxo6. This gene encodes a transcription factor. Its functions include regulation of Hippo signaling and growth of the craniofacial complex [71].

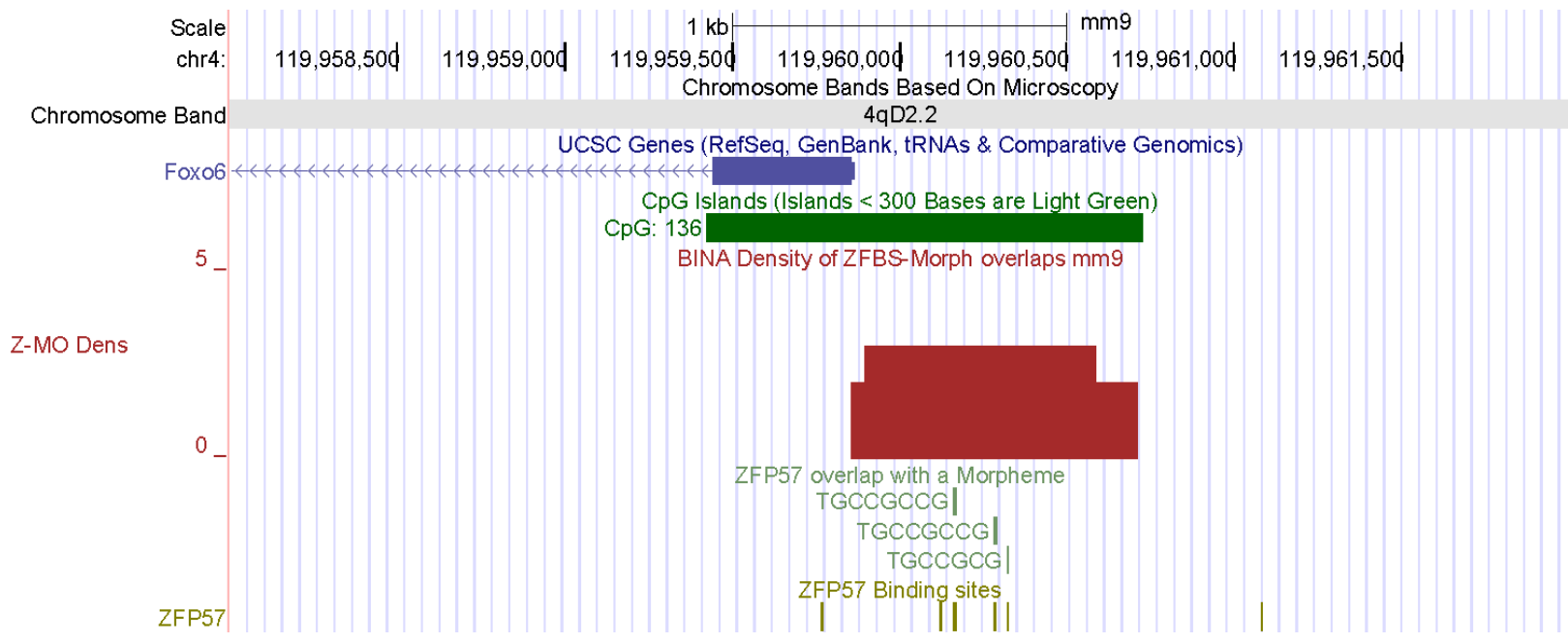

Fig. S23. The position of a candidate ICR for Pou3f1. A reduction in Pou3f1 expression induced apoptosis of cultured germ cells [72]. Biochemical studies localized POU3F1 to spermatogonia of prepubertal and adult testes [72].

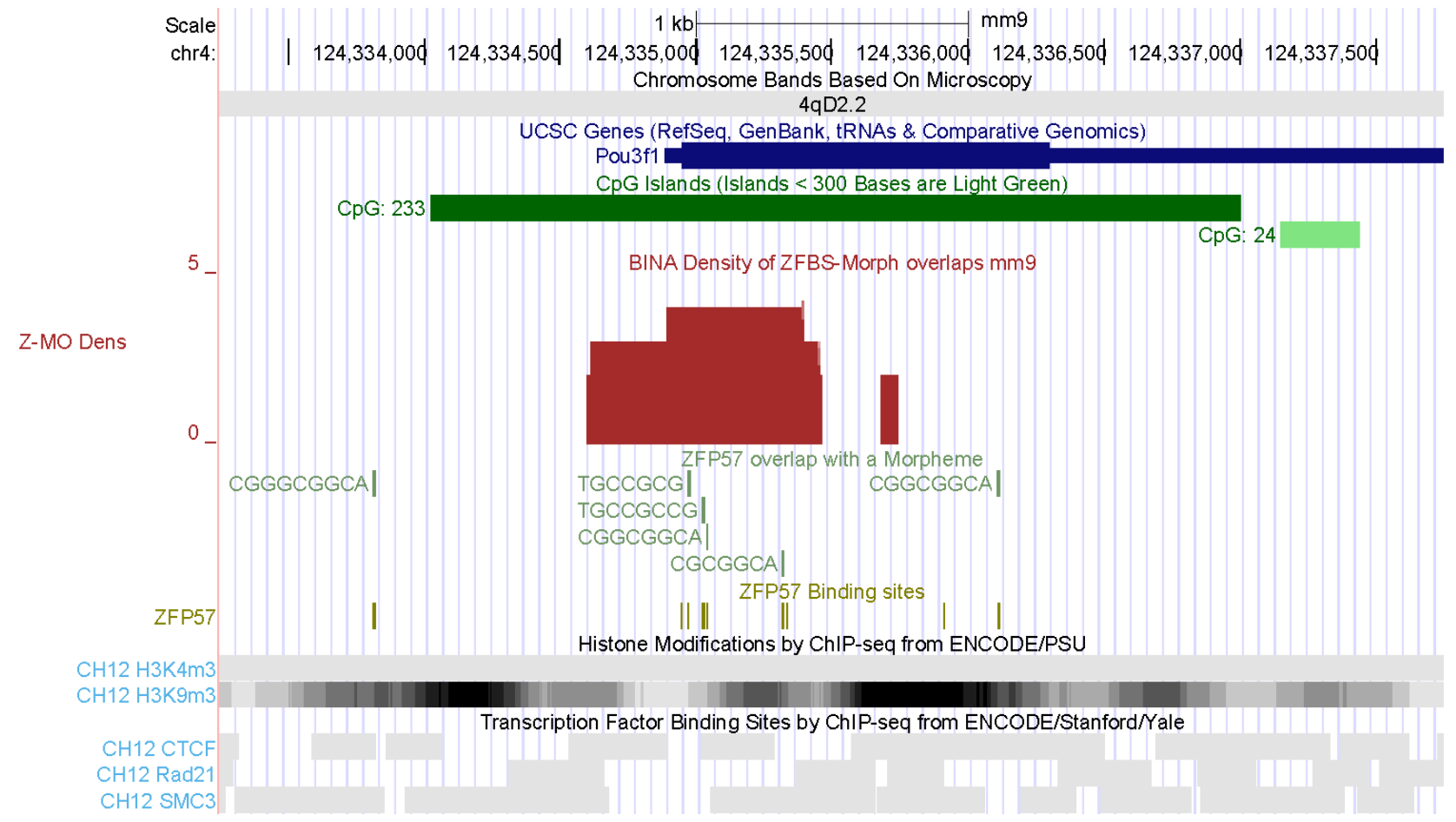


bioRxiv preprint doi: https://doi.org/10.1101/780551; this version posted September 24, 2019. The copyright holder for this preprint (which was not certified by peer review) is the author/funder. All rights reserved. No reuse allowed without permission.

Fig. S24. The position of a candidate ICR for the longest Mgea5 transcript. This gene encodes an enzyme that influences folliculogenesis [73].

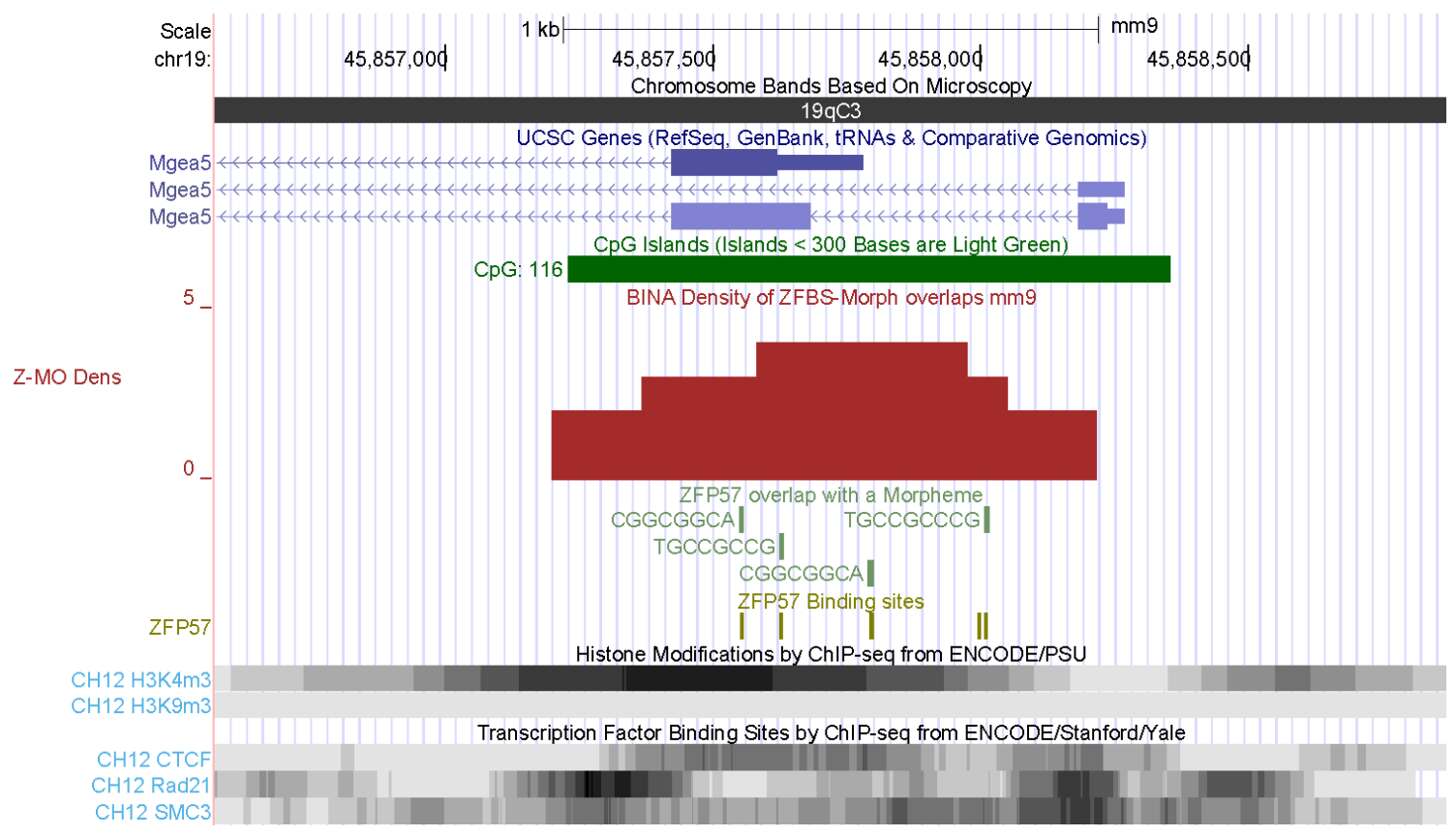

Fig. S25. The position of a candidate mapping to Cnnm1. Expression of this gene is associated with cell cycle and differentiation in spermatogenic cells in mouse testis [74].

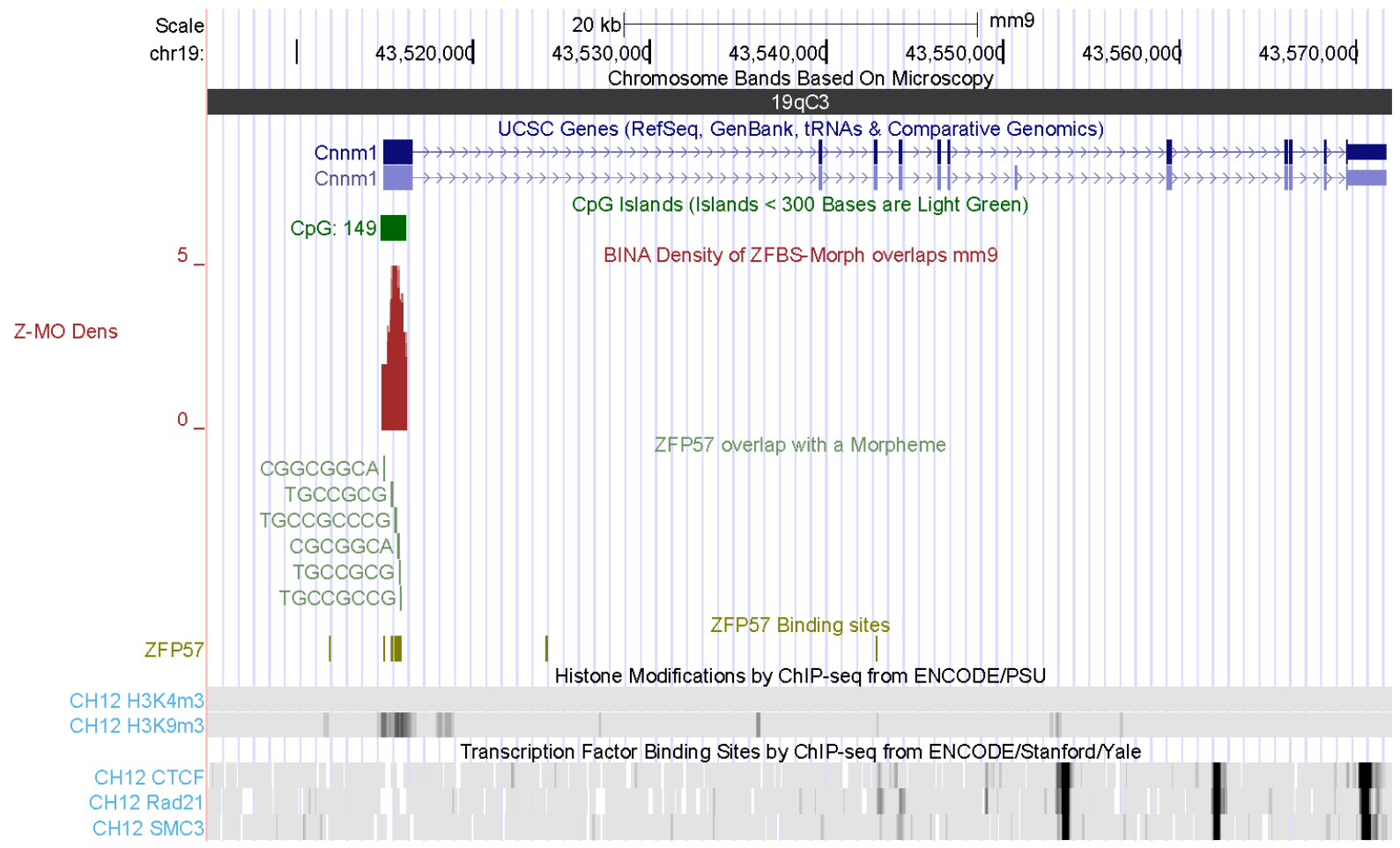


Fig. S26. The position of a candidate ICR mapping to Chd5. This gene encodes an enzyme that mediates histone-to-protamine replacement and thus impacts the cascade of molecular events underlying chromatin remodeling during spermatogenesis [75].

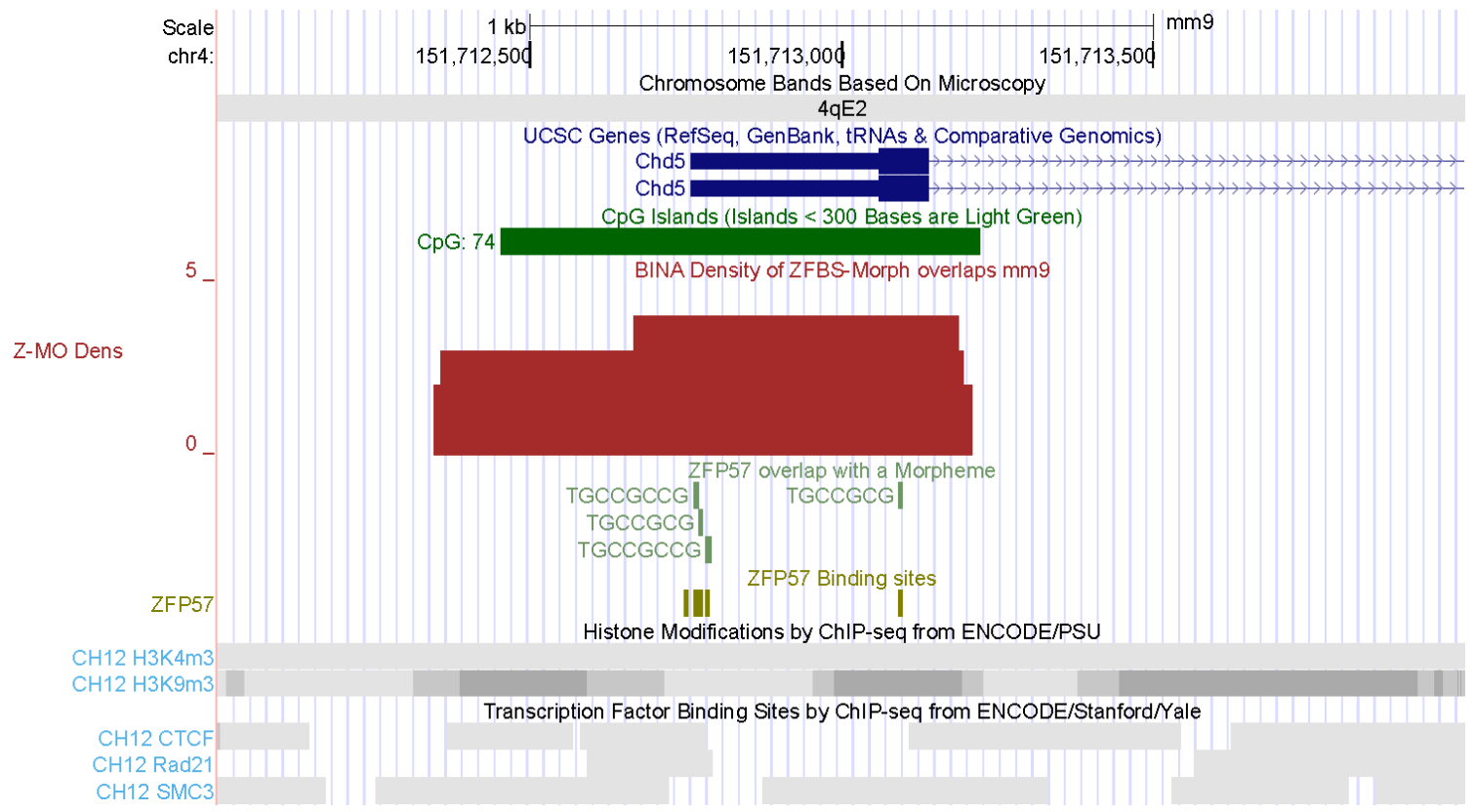

Fig. S27. A candidate ICR mapping to longest transcripts of a gene (BC051142) expressed in testes. The corresponding density peak maps to a chromatin boundary.

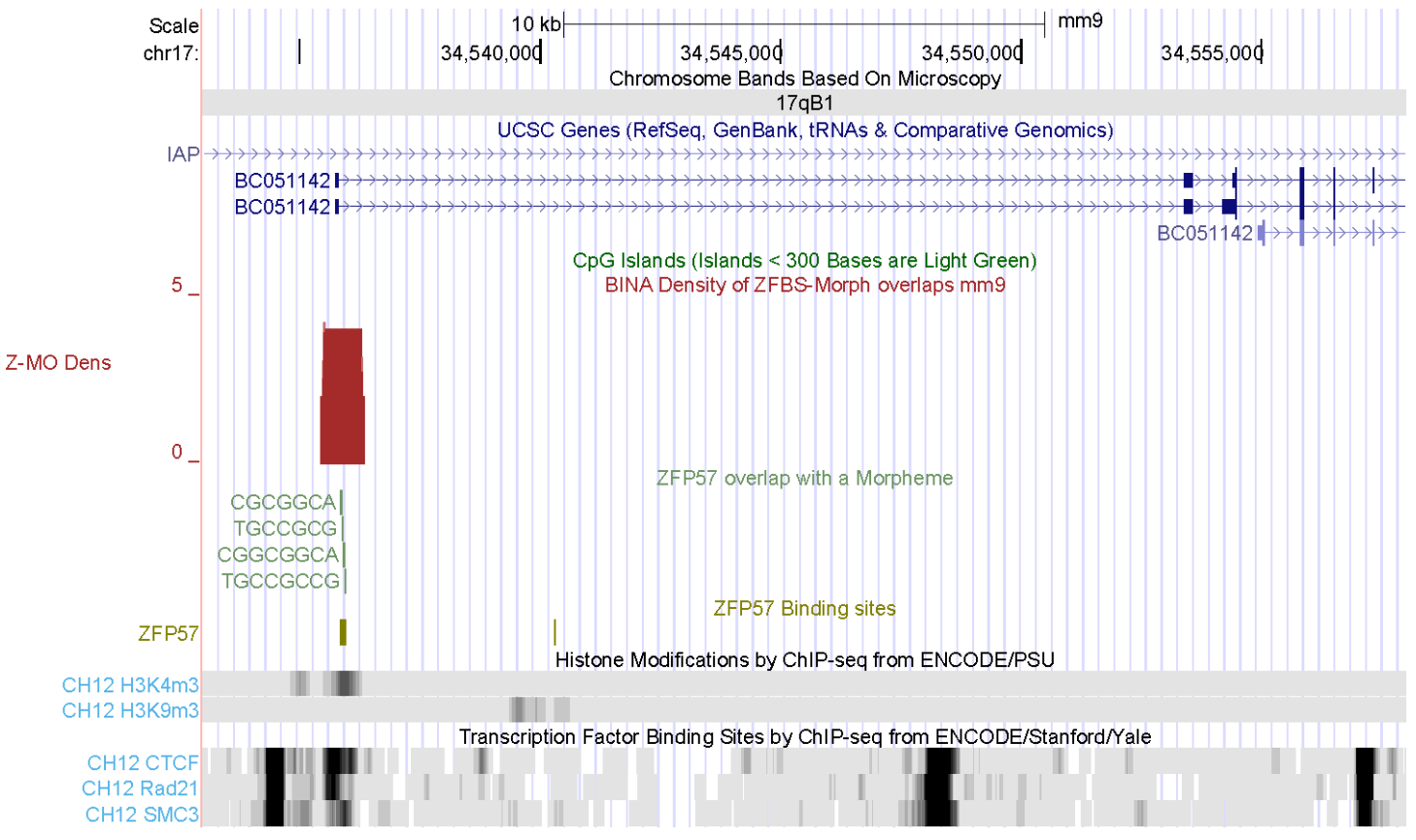




\section{REFERENCES}

1. Barton SC, Surani MA, Norris ML: Role of paternal and maternal genomes in mouse development. Nature 1984, 311(5984):374-376.

2. McGrath J, Solter D: Completion of mouse embryogenesis requires both the maternal and paternal genomes. Cell 1984, 37(1):179-183.

3. Surani MA, Barton SC, Norris ML: Development of reconstituted mouse eggs suggests imprinting of the genome during gametogenesis. Nature 1984, 308(5959):548-550.

4. Barlow DP, Bartolomei MS: Genomic imprinting in mammals. Cold Spring Harbor perspectives in biology 2014, 6(2).

5. Ferguson-Smith AC, Bourc'his D: The discovery and importance of genomic imprinting. Elife 2018, 7.

6. Strogantsev R, Ferguson-Smith AC: Proteins involved in establishment and maintenance of imprinted methylation marks. Brief Funct Genomics 2012, 11(3):227-239.

7. Stewart KR, Veselovska L, Kelsey G: Establishment and functions of DNA methylation in the germline. Epigenomics 2016, 8(10):1399-1413.

8. Holz-Schietinger $C$, Reich NO: The inherent processivity of the human de novo methyltransferase $3 \mathrm{~A}$ (DNMT3A) is enhanced by DNMT3L. J Biol Chem 2010, 285(38):29091-29100.

9. Bina M, Wyss P, Novorolsky E, Zulkelfi N, Xue J, Price R, Fay M, Gutmann Z, Fogler B, Wang D: Discovery of MLL1 binding units, their localization to CpG Islands, and their potential function in mitotic chromatin. BMC Genomics 2013, 14:927.

10. Birke M, Schreiner S, Garcia-Cuellar MP, Mahr K, Titgemeyer F, Slany RK: The MT domain of the protooncoprotein MLL binds to CpG-containing DNA and discriminates against methylation. Nucleic Acids Res 2002, 30(4):958-965.

11. Bach C, Mueller D, Buhl S, Garcia-Cuellar MP, Slany RK: Alterations of the CxxC domain preclude oncogenic activation of mixed-lineage leukemia 2. Oncogene 2009, 28(6):815-823.

12. Ruthenburg AJ, Allis CD, Wysocka J: Methylation of lysine 4 on histone H3: intricacy of writing and reading a single epigenetic mark. Mol Cell 2007, 25(1):15-30.

13. Bina M, Wyss P, Song XC: Datasets on the genomic positions of the MLL1 morphemes, the ZFP57 binding site, and ZFBS-Morph overlaps in the build $\mathrm{mm} 9$ of the mouse genome. Data Brief 2017, 13:202-207.

14. Branciamore S, Chen ZX, Riggs AD, Rodin SN: CpG island clusters and pro-epigenetic selection for CpGs in protein-coding exons of HOX and other transcription factors. Proc Natl Acad Sci U S A 2010, 107(35):15485-15490.

15. Bina M, Wyss P: Impact of the MLL1 morphemes on codon utilization and preservation in CpG Islands. Biopolymers 2015, 103(9):480-490.

16. Bina M: Imprinted control regions include composite DNA elements consisting of the ZFP57 binding site overlapping MLL1 morphemes. Genomics 2017.

17. Quenneville S, Verde G, Corsinotti A, Kapopoulou A, Jakobsson J, Offner S, Baglivo I, Pedone PV, Grimaldi G, Riccio A et al: In embryonic stem cells, ZFP57/KAP1 recognize a methylated hexanucleotide to affect chromatin and DNA methylation of imprinting control regions. Mol Cell 2011, 44(3):361-372.

18. Bird AP: DNA methylation and the frequency of CpG in animal DNA. Nucleic Acids Res 1980, 8(7):1499-1504.

19. Bina M: Discovering candidate imprinted genes and Imprinting Control Regions in the human genome. bioRxiv 2019, https://www.biorxiv.org/content/10.1101/678151v1.

20. Hark AT, Schoenherr CJ, Katz DJ, Ingram RS, Levorse JM, Tilghman SM: CTCF mediates methylationsensitive enhancer-blocking activity at the H19/Igf2 locus. Nature 2000, 405(6785):486-489. 
21. Bell AC, Felsenfeld G: Methylation of a CTCF-dependent boundary controls imprinted expression of the Igf2 gene. Nature 2000, 405(6785):482-485.

22. Shi H, Strogantsev R, Takahashi N, Kazachenka A, Lorincz MC, Hemberger M, Ferguson-Smith AC: ZFP57 regulation of transposable elements and gene expression within and beyond imprinted domains. Epigenetics Chromatin 2019, 12(1):49.

23. Zhang T, Li Y, Li H, Ma XS, Ouyang YC, Hou Y, Schatten H, Sun QY: RNA-associated protein LSM family member 14 controls oocyte meiotic maturation through regulating mRNA pools. The Journal of reproduction and development 2017, 63(4):383-388.

24. van Soest $S$, Westerveld A, de Jong PT, Bleeker-Wagemakers EM, Bergen AA: Retinitis pigmentosa: defined from a molecular point of view. Surv Ophthalmol 1999, 43(4):321-334.

25. Mortimer SE, Hedstrom L: Autosomal dominant retinitis pigmentosa mutations in inosine 5'monophosphate dehydrogenase type I disrupt nucleic acid binding. Biochem J 2005, 390(Pt 1):41-47.

26. Sabour D, Srinivasan SP, Rohani S, Wagh V, Gaspar JA, Panek D, Ardestani MA, Doss MX, Riet N, Abken $\mathrm{H}$ et al: STRIP2 Is Indispensable for the Onset of Embryonic Stem Cell Differentiation. Mol Ther Methods Clin Dev 2017, 5:116-129.

27. Yazbek SN, Buchner DA, Geisinger JM, Burrage LC, Spiezio SH, Zentner GE, Hsieh CW, Scacheri PC, Croniger CM, Nadeau JH: Deep congenic analysis identifies many strong, context-dependent QTLs, one of which, Slc35b4, regulates obesity and glucose homeostasis. Genome Res 2011, 21(7):10651073.

28. Smith RJ, Arnaud P, Konfortova G, Dean WL, Beechey CV, Kelsey G: The mouse Zac1 locus: basis for imprinting and comparison with human ZAC. Gene 2002, 292(1-2):101-112.

29. Karemaker ID, Vermeulen M: ZBTB2 reads unmethylated CpG island promoters and regulates embryonic stem cell differentiation. EMBO reports 2018, 19(4).

30. Antonin W, Holroyd C, Fasshauer D, Pabst S, Von Mollard GF, Jahn R: A SNARE complex mediating fusion of late endosomes defines conserved properties of SNARE structure and function. EMBO J 2000, 19(23):6453-6464.

31. Nomura N, Zhao MJ, Nagase T, Maekawa T, Ishizaki R, Tabata S, Ishii S: HIV-EP2, a new member of the gene family encoding the human immunodeficiency virus type 1 enhancer-binding protein. Comparison with HIV-EP1/PRDII-BF1/MBP-1. J Biol Chem 1991, 266(13):8590-8594.

32. Takao K, Kobayashi K, Hagihara H, Ohira K, Shoji H, Hattori S, Koshimizu H, Umemori J, Toyama K, Nakamura HK et al: Deficiency of schnurri-2, an MHC enhancer binding protein, induces mild chronic inflammation in the brain and confers molecular, neuronal, and behavioral phenotypes related to schizophrenia. Neuropsychopharmacology 2013, 38(8):1409-1425.

33. Lopes Floro K, Artap ST, Preis JI, Fatkin D, Chapman G, Furtado MB, Harvey RP, Hamada H, Sparrow DB, Dunwoodie SL: Loss of Cited2 causes congenital heart disease by perturbing left-right patterning of the body axis. Hum Mol Genet 2011, 20(6):1097-1110.

34. Wutz A, Smrzka OW, Schweifer N, Schellander K, Wagner EF, Barlow DP: Imprinted expression of the Igf2r gene depends on an intronic CpG island. Nature 1997, 389(6652):745-749.

35. Braidotti G, Baubec T, Pauler F, Seidl C, Smrzka O, Stricker S, Yotova I, Barlow DP: The Air noncoding RNA: an imprinted cis-silencing transcript. Cold Spring Harb Symp Quant Biol 2004, 69:55-66.

36. Verona RI, Mann MR, Bartolomei MS: Genomic imprinting: intricacies of epigenetic regulation in clusters. Annu Rev Cell Dev Biol 2003, 19:237-259.

37. Haeussler M, Zweig AS, Tyner C, Speir ML, Rosenbloom KR, Raney BJ, Lee CM, Lee BT, Hinrichs AS, Gonzalez JN et al: The UCSC Genome Browser database: 2019 update. Nucleic Acids Res 2019, 47(D1):D853-D858.

38. Zweig AS, Karolchik D, Kuhn RM, Haussler D, Kent WJ: UCSC genome browser tutorial. Genomics 2008, 92(2):75-84. 
39. Bina M: The genome browser at UCSC for locating genes, and much more! Molecular biotechnology 2008, 38(3):269-275.

40. Gigante S, Gouil Q, Lucattini A, Keniry A, Beck T, Tinning M, Gordon L, Woodruff C, Speed TP, Blewitt $\mathrm{ME}$ et al: Using long-read sequencing to detect imprinted DNA methylation. Nucleic Acids Res 2019.

41. Kidder BL, Hu G, Zhao K: KDM5B focuses H3K4 methylation near promoters and enhancers during embryonic stem cell self-renewal and differentiation. Genome Biol 2014, 15(2):R32.

42. Li X, Ito M, Zhou F, Youngson N, Zuo X, Leder P, Ferguson-Smith AC: A maternal-zygotic effect gene, Zfp57, maintains both maternal and paternal imprints. Dev Cell 2008, 15(4):547-557.

43. Agata $Y$, Matsuda E, Shimizu A: Two novel Kruppel-associated box-containing zinc-finger proteins, KRAZ1 and KRAZ2, repress transcription through functional interaction with the corepressor KAP-1 (TIF1beta/KRIP-1). J Biol Chem 1999, 274(23):16412-16422.

44. Takahashi N, Coluccio A, Thorball CW, Planet E, Shi H, Offner S, Turelli P, Imbeault M, Ferguson-Smith AC, Trono D: ZNF445 is a primary regulator of genomic imprinting. Genes Dev 2019, 33(1-2):49-54.

45. Artzt $\mathrm{K}$, Shin HS, Bennett D: Gene mapping within the $\mathrm{T} / \mathrm{t}$ complex of the mouse. II. Anomalous position of the $\mathbf{H - 2}$ complex in t haplotypes. Cell 1982, 28(3):471-476.

46. Lyon MF: Transmission ratio distortion in mice. Annual review of genetics 2003, 37:393-408.

47. Olds-Clarke P: Models for male infertility: the thaplotypes. Rev Reprod 1997, 2(3):157-164.

48. Lader $E, H a$ HS, O'Neill M, Artzt $K$, Bennett $D$ : tctex-1: a candidate gene family for a mouse $t$ complex sterility locus. Cell 1989, 58(5):969-979.

49. King SM: The dynein microtubule motor. Biochimica et biophysica acta 2000, 1496(1):60-75.

50. Kagitani F, Kuroiwa Y, Wakana S, Shiroishi T, Miyoshi N, Kobayashi S, Nishida M, Kohda T, KanekoIshino T, Ishino F: Peg5/Neuronatin is an imprinted gene located on sub-distal chromosome $\mathbf{2}$ in the mouse. Nucleic Acids Res 1997, 25(17):3428-3432.

51. Evans HK, Wylie AA, Murphy SK, Jirtle RL: The neuronatin gene resides in a "micro-imprinted" domain on human chromosome 20q11.2. Genomics 2001, 77(1-2):99-104.

52. John RM, Aparicio SA, Ainscough JF, Arney KL, Khosla S, Hawker K, Hilton KJ, Barton SC, Surani MA: Imprinted expression of neuronatin from modified BAC transgenes reveals regulation by distinct and distant enhancers. Dev Biol 2001, 236(2):387-399.

53. DeVeale B, van der Kooy D, Babak T: Critical evaluation of imprinted gene expression by RNA-Seq: a new perspective. PLoS Genet 2012, 8(3):e1002600.

54. Kasai S, Chuma S, Motoyama N, Nakatsuji N: Haploinsufficiency of Bcl-x leads to male-specific defects in fetal germ cells: differential regulation of germ cell apoptosis between the sexes. Dev Biol 2003, 264(1):202-216.

55. Perez JD, Rubinstein ND, Fernandez DE, Santoro SW, Needleman LA, Ho-Shing O, Choi JJ, Zirlinger M, Chen SK, Liu JS et al: Quantitative and functional interrogation of parent-of-origin allelic expression biases in the brain. Elife 2015, 4:e07860.

56. Zheng X, Xu C, Smith AO, Stratman AN, Zou Z, Kleaveland B, Yuan L, Didiku C, Sen A, Liu X et al: Dynamic regulation of the cerebral cavernous malformation pathway controls vascular stability and growth. Dev Cell 2012, 23(2):342-355.

57. Baranoski JF, Kalani MY, Przybylowski CJ, Zabramski JM: Cerebral Cavernous Malformations: Review of the Genetic and Protein-Protein Interactions Resulting in Disease Pathogenesis. Front Surg 2016, 3:60.

58. Price M, Lazzaro D, Pohl T, Mattei MG, Ruther U, Olivo JC, Duboule D, Di Lauro R: Regional expression of the homeobox gene Nkx-2.2 in the developing mammalian forebrain. Neuron 1992, 8(2):241-255.

59. Choi JD, Underkoffler LA, Wood AJ, Collins JN, Williams PT, Golden JA, Schuster EF, Jr., Loomes KM, Oakey RJ: A novel variant of Inpp5f is imprinted in brain, and its expression is correlated with differential methylation of an internal CpG island. Mol Cell Biol 2005, 25(13):5514-5522. 
60. Kosaki K, Kosaki R, Craigen WJ, Matsuo N: Isoform-specific imprinting of the human PEG1/MEST gene. American journal of human genetics 2000, 66(1):309-312.

61. Mouse EC, Stamatoyannopoulos JA, Snyder M, Hardison R, Ren B, Gingeras T, Gilbert DM, Groudine M, Bender M, Kaul R et al: An encyclopedia of mouse DNA elements (Mouse ENCODE). Genome Biol 2012, 13(8):418.

62. Moody SA, LaMantia AS: Transcriptional regulation of cranial sensory placode development. Curr Top Dev Biol 2015, 111:301-350.

63. Sarkar AA, Nuwayhid SJ, Maynard T, Ghandchi F, Hill JT, Lamantia AS, Zohn IE: Hectd1 is required for development of the junctional zone of the placenta. Dev Biol 2014, 392(2):368-380.

64. Kale J, Osterlund EJ, Andrews DW: BCL-2 family proteins: changing partners in the dance towards death. Cell Death Differ 2018, 25(1):65-80.

65. Bina M, Demmon S, Pares-Matos El: Syndromes associated with Homo sapiens pol II regulatory genes. Prog Nucleic Acid Res Mol Biol 2000, 64:171-219.

66. Heng YH, Zhou B, Harris L, Harvey T, Smith A, Horne E, Martynoga B, Andersen J, Achimastou A, Cato K et al: NFIX Regulates Proliferation and Migration Within the Murine SVZ Neurogenic Niche. Cerebral cortex 2015, 25(10):3758-3778.

67. Oyama T, Harigaya K, Sasaki N, Okamura Y, Kokubo H, Saga Y, Hozumi K, Suganami A, Tamura Y, Nagase T et al: Mastermind-like 1 (MamL1) and mastermind-like 3 (MamL3) are essential for Notch signaling in vivo. Development 2011, 138(23):5235-5246.

68. Dominguez MH, Ayoub AE, Rakic P: POU-III transcription factors (Brn1, Brn2, and Oct6) influence neurogenesis, molecular identity, and migratory destination of upper-layer cells of the cerebral cortex. Cerebral cortex 2013, 23(11):2632-2643.

69. Herr W, Cleary MA: The POU domain: versatility in transcriptional regulation by a flexible two-in-one DNA-binding domain. Genes Dev 1995, 9(14):1679-1693.

70. Kaufmann E, Knochel W: Five years on the wings of fork head. Mechanisms of development 1996, 57(1):3-20.

71. Sun Z, da Fontoura CSG, Moreno M, Holton NE, Sweat M, Sweat Y, Lee MK, Arbon J, Bidlack FB, Thedens DR et al: FoxO6 regulates Hippo signaling and growth of the craniofacial complex. PLOS Genet 2018, 14(10):e1007675.

72. Wu X, Oatley JM, Oatley MJ, Kaucher AV, Avarbock MR, Brinster RL: The POU domain transcription factor POU3F1 is an important intrinsic regulator of GDNF-induced survival and self-renewal of mouse spermatogonial stem cells. Biol Reprod 2010, 82(6):1103-1111.

73. Hatzirodos N, Irving-Rodgers HF, Hummitzsch K, Harland ML, Morris SE, Rodgers RJ: Transcriptome profiling of granulosa cells of bovine ovarian follicles during growth from small to large antral sizes. BMC Genomics 2014, 15:24.

74. Chandran U, Indu S, Kumar AT, Devi AN, Khan I, Srivastava D, Kumar PG: Expression of Cnnm1 and Its Association with Stemness, Cell Cycle, and Differentiation in Spermatogenic Cells in Mouse Testis. Biol Reprod 2016, 95(1):7.

75. Li W, Wu J, Kim SY, Zhao M, Hearn SA, Zhang MQ, Meistrich ML, Mills AA: Chd5 orchestrates chromatin remodelling during sperm development. Nature communications 2014, 5:3812.

76. Li W, Mills AA: Packing for the journey: CHD5 remodels the genome. Cell Cycle 2014, 13(12):18331834.

77. Miyata H, Castaneda JM, Fujihara Y, Yu Z, Archambeault DR, Isotani A, Kiyozumi D, Kriseman ML, Mashiko $D$, Matsumura $T$ et al: Genome engineering uncovers 54 evolutionarily conserved and testisenriched genes that are not required for male fertility in mice. Proc Natl Acad Sci U S A 2016, 113(28):7704-7710.

78. Kelsey G, Bartolomei MS: Imprinted genes ... and the number is? PLoS Genet 2012, 8(3):e1002601. 
79. Wood AJ, Bourc'his D, Bestor TH, Oakey RJ: Allele-specific demethylation at an imprinted mammalian promoter. Nucleic Acids Res 2007, 35(20):7031-7039.

80. Abdollahi A: LOT1 (ZAC1/PLAGL1) and its family members: mechanisms and functions. Journal of cellular physiology 2007, 210(1):16-25.

81. Zuin J, Dixon JR, van der Reijden MI, Ye Z, Kolovos P, Brouwer RW, van de Corput MP, van de Werken HJ, Knoch TA, van IWF et al: Cohesin and CTCF differentially affect chromatin architecture and gene expression in human cells. Proc Natl Acad Sci U S A 2014, 111(3):996-1001.

82. Henckel A, Arnaud P: Genome-wide identification of new imprinted genes. Brief Funct Genomics 2010, 9(4):304-314.

83. Ciccone DN, Su H, Hevi S, Gay F, Lei H, Bajko J, Xu G, Li E, Chen T: KDM1B is a histone H3K4 demethylase required to establish maternal genomic imprints. Nature 2009, 461(7262):415-418.

84. Heng YH, McLeay RC, Harvey TJ, Smith AG, Barry G, Cato K, Plachez C, Little E, Mason S, Dixon C et al: NFIX regulates neural progenitor cell differentiation during hippocampal morphogenesis. Cerebral cortex 2014, 24(1):261-279.

85. Love DC, Krause MW, Hanover JA: O-GICNAc cycling: emerging roles in development and epigenetics. Seminars in cell \& developmental biology 2010, 21(6):646-654.

86. Bina $\mathrm{M}$, Wyss PJ, Song XC: The positions of ZFBS and ZFBS-Morph overlaps in the build mm9 of the mouse genome. Purdue University Research Repository 2017, https://purr.purdue.edu/publications/2473/1.

87. Bina M, Wyss PJ, Song XC: Density of ZFBS-Morph overlaps in the build $\mathbf{m m} 9$ of the mouse genome. Purdue University Research Repository 2018, https://purr.purdue.edu/publications/2961/1.

88. Bina $M$, Wyss $P$, Wang D, Song X: Localization of MLL1 morphemes in mouse $\mathbf{m m} 9$ genomic DNA. Purdue University Research Repository 2014.

89. Bina M, Wyss PJ, Song XC: The positions of ZFBS and ZFBS-Morph overlaps in the build hg19 of the human genome. Purdue University Research Repository 2019, https://purr.purdue.edu/publications/3208/1.

90. Bina M, Wyss PJ, Song XC: Density of ZFBS-Morph overlaps in the build hg19 of the human genome. . Purdue University Research Repository 2019, https://purr.purdue.edu/publications/2967/1.

91. Bina M, Wyss PJ, Wang D, Zulkefl N, Novorolsky E: Position of MLL1 morphemes in human genomic DNA. Purdue University Research Repository 2014, https://purr.purdue.edu/publications/1639/1.

92. Yue F, Cheng Y, Breschi A, Vierstra J, Wu W, Ryba T, Sandstrom R, Ma Z, Davis C, Pope BD et al: A comparative encyclopedia of DNA elements in the mouse genome. Nature 2014, 515(7527):355-364.

93. Fox HS, Martin GR, Lyon MF, Herrmann B, Frischauf AM, Lehrach H, Silver LM: Molecular probes define different regions of the mouse $t$ complex. Cell 1985, 40(1):63-69.

94. Ozaki H, Nakamura K, Funahashi J, Ikeda K, Yamada G, Tokano H, Okamura HO, Kitamura K, Muto S, Kotaki $\mathrm{H}$ et al: Six1 controls patterning of the mouse otic vesicle. Development 2004, 131(3):551-562.

95. Herndon MK, Law NC, Donaubauer EM, Kyriss B, Hunzicker-Dunn M: Forkhead box O member FOxO1 regulates the majority of follicle-stimulating hormone responsive genes in ovarian granulosa cells. Molecular and cellular endocrinology 2016, 434:116-126.

96. Clark BS, Blackshaw S: Understanding the Role of IncRNAs in Nervous System Development. Advances in experimental medicine and biology 2017, 1008:253-282.

97. Jansen JG, Langerak P, Tsaalbi-Shtylik A, van den Berk P, Jacobs $H$, de Wind N: Strand-biased defect in C/G transversions in hypermutating immunoglobulin genes in Rev1-deficient mice. J Exp Med 2006, 203(2):319-323. 